\title{
Giant radio galaxies in the LOFAR Two-metre Sky Survey
}

\section{Radio and environmental properties ${ }^{\star}$}

\author{
P. Dabhade ${ }^{1,2}$, H. J. A. Röttgering ${ }^{1}$, J. Bagchi ${ }^{2}$, T. W. Shimwell ${ }^{1,3}$, M. J. Hardcastle ${ }^{4}$, S. Sankhyayan ${ }^{5}$, R. Morganti ${ }^{3,6}$, \\ M. Jamrozy ${ }^{7}$, A. Shulevski ${ }^{8}$, and K. J. Duncan ${ }^{1}$
}

${ }^{1}$ Leiden Observatory, Leiden University, PO Box 9513, 2300 RA Leiden, The Netherlands e-mail: pratik@strw.leidenuniv.nl

2 Inter University Centre for Astronomy and Astrophysics (IUCAA), Pune 411007, India

3 ASTRON, The Netherlands Institute for Radio Astronomy, Postbus 2, 7990 AA Dwingeloo, The Netherlands

${ }^{4}$ Centre for Astrophysics Research, School of Physics, Astronomy and Mathematics, University of Hertfordshire, College Lane, Hatfield AL10 9AB, UK

5 Indian Institute of Science Education and Research (IISER), Dr. Homi Bhabha Road, Pashan, Pune 411008, India

${ }^{6}$ Kapteyn Astronomical Institute, University of Groningen, PO Box 800, 9700 AV Groningen, The Netherlands

7 Astronomical Observatory, Jagiellonian University, ul. Orla 171, 30-244 Kraków, Poland

8 Anton Pannekoek Institute for Astronomy, University of Amsterdam, Postbus 94249, 1090 GE Amsterdam, The Netherlands

Received 31 March 2019 / Accepted 3 December 2019

\begin{abstract}
Giant radio galaxies (GRGs) are a subclass of radio galaxies, which have grown to megaparsec scales. GRGs are much rarer than normal-sized radio galaxies $(<0.7 \mathrm{Mpc})$ and the reason for their gigantic sizes is still debated. Here, we report on the biggest sample of GRGs identified to date. These objects were found in the LOFAR Two-metre Sky Survey first data release images, which cover a $424 \mathrm{deg}^{2}$ region. Of the 239 GRGs found, 225 are new discoveries. The GRGs in our sample have sizes ranging from $0.7 \mathrm{Mpc}$ to $3.5 \mathrm{Mpc}$ and have redshifts $(z)$ between 0.1 and 2.3. Seven GRGs have sizes above $2 \mathrm{Mpc}$ and one has a size of $\sim 3.5 \mathrm{Mpc}$. The sample contains 40 GRGs hosted by spectroscopically confirmed quasars. Here, we present the search techniques employed and the resulting catalogue of the newly discovered large sample of GRGs along with their radio properties. In this paper, we also show for the first time that the spectral index of GRGs is similar to that of normal-sized radio galaxies, indicating that most of the GRG population is not dead or is not similar to a remnant-type radio galaxy. We find that 20 out of 239 GRGs in our sample are located at the centres of clusters and we present our analysis on their cluster environment and radio morphology.
\end{abstract}

Key words. galaxies: jets - galaxies: active - radio continuum: galaxies - quasars: general

\section{Introduction}

A radio galaxy $(\mathrm{RG})$ normally contains a radio core, jets, and lobes powered by an active galactic nucleus (AGN). A RG that has grown to $\mathrm{Mpc}$ scales is traditionally defined as a giant radio galaxy (GRG; Willis et al. 1974; Ishwara-Chandra \& Saikia 1999). Here, the total size is defined as the largest angular separation between the end of the two radio lobes. This subclass of radio galaxies (RGs) is among the largest single structures known in the Universe along with the cluster radio relics (Rottgering et al. 1997; Bagchi et al. 2006; van Weeren et al. 2011). Born in the active nucleus of a galaxy or a quasar, RGs and quasars eject collimated and bipolar relativistic jets (Lynden-Bell 1969; Begelman et al. 1984). The driving engine for these jets is an accreting supermassive black hole (SMBH) with a typical mass of $10^{8}-10^{10} M_{\odot}$. SMBHs that drive powerful jets reside in elliptical galaxies and only a handful are found in spiral galaxies (Hota et al. 2011; Bagchi et al. 2014).

Morphologically, RGs have been historically divided into two classes, Fanaroff-Riley type I (FR-I) and Fanaroff-Riley type II (FR-II). The lower radio luminosity FR-I sources have

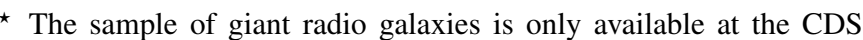
via anonymous ftp to cdsarc. u-strasbg. fr (130.79.128.5) or via http://cdsarc.u-strasbg.fr/viz-bin/cat/J/A+A/635/A5
}

their brightest regions closer to the nucleus and their jets fade with distance from the core. For the more powerful FR-II RGs (Fanaroff \& Riley 1974), the jet remains relativistic all the way from the central AGN to the hotspots in lobes.

For all RGs the ejection of a collimated jet is dependent on the availability of fuel. Assuming GRGs grow to an enormous size due to a prolonged period activity, this would require either an unusually large reservoir of fuel or a very efficient jet formation mechanism.

Radio galaxies were first discovered about six decades ago (Jennison \& Das Gupta 1953) and since then hundreds of thousands of RGs have been found. In contrast to the large number of radio galaxies, only 350 GRGs (Dabhade et al. 2017; Kuźmicz et al. 2018, and references therein) have been found of which only a small fraction has been studied in detail. These giants, when associated with quasars as their AGN, are called giant radio quasars (GRQs) and only around 70 GRQs are known so far (Kuźmiczet al. 2018). The term "GRQ" is used here to emphasise the fact that the GRG has quasar as the powering AGN.

The hypotheses proposed to explain the enormous sizes of GRGs are not mutually exclusive and include:

1. GRGs possess exceptionally powerful radio jets, when compared to normal RGs, and these provide the necessary thrust to reach Mpc scales (Wiita et al. 1989). 
2. GRGs are very old RGs and have had sufficient time to expand over large distances (Subrahmanyan et al. 1996).

3. GRGs grow in low density environments, (Mack et al. 1998; Malarecki et al. 2015; Saripalli \& Malarecki 2015) enabling them to grow comparatively fast.

None of the above hypotheses have been tested using large uniform samples of GRGs. In smaller samples, contradictory results have been found. For example, Mack et al. (1998) found evidence that the ages of GRGs in their sample are similar to that of normal-sized radio galaxies (NRGs), which is contradictory to the second above mentioned point. Also, point three has been contradicted by the findings of Komberg \& Pashchenko (2009) and Dabhade et al. (2017) who have reported that a number of GRGs are located in cluster environments.

The exceptionally large lobes of GRGs make them excellent laboratories for studying the evolution of the particle and magnetic field energy density and the acceleration of high-energy cosmic rays. They can also be used to probe large-scale environments (Kronberg et al. 2004; Safouris et al. 2009; Malarecki et al. 2015; Isobe \& Koyama 2015).

Until the mid-1990s, GRGs were mostly discovered serendipitously. Only after the advent of deep and large sky radio surveys, such as the Faint Images of the Radio Sky at Twenty-cm (FIRST; Becker et al. 1995), NRAO VLA Sky Survey (NVSS; Condon et al. 1998), Westerbork Northern Sky Survey (WENSS; Rengelink et al. 1997), and Sydney University Molonglo Sky Survey (SUMSS; Bock et al. 1999), were systematic searches for GRGs carried out. Lara et al. (2001), Machalski et al. (2001), and Dabhade et al. (2017) searched the higher frequency survey, such as NVSS (1400 MHz) for GRGs, and Saripalli et al. (2005) used SUMSS, whereas Schoenmakers et al. (2001) used lower frequency surveys, such as WENSS (327 MHz).

The lobes of the GRGs have steep spectral indices and hence are bright at low radio frequencies. Cotter et al. (1996) made a sample of GRGs by using the $151 \mathrm{MHz} 7 \mathrm{C}$ survey (McGilchrist $\&$ Riley 1990). The 7C survey has a low resolution of $70 \times 70$ $\operatorname{cosec}(\delta) \operatorname{arcsec}^{2}$ and a noise level $\sim 15 \mathrm{mJy}^{-1}$ beam $^{-1}(1 \sigma)$ and as a consequence contained only a few GRGs. The WENSS has better resolution $\left(54 \times 54 \operatorname{cosec}(\delta) \operatorname{arcsec}^{2}\right)$ and better sensitivity (rms noise $(1 \sigma) \sim 3 \mathrm{mJy}^{-1}$ beam $^{-1}$ ) and also covers a somewhat larger area $\sim 8100 \mathrm{deg}^{2}$ (7C survey $\sim 5580 \mathrm{deg}^{2}$ ). This enabled Schoenmakers et al. (2001) to compile a large sample of 47 GRGs from the WENSS.

In recent years, four large low frequency surveys have been carried out, namely: the $119-158 \mathrm{MHz}$ Multifrequency Snapshot Sky Survey (MSSS; Heald et al. 2015), the $150 \mathrm{MHz}$ TIFR GMRT SKY SURVEY- Alternative data release-1 (TGSSADR1; Intema et al. 2017), the $72-231 \mathrm{MHz}$ GaLactic and Extragalactic All-sky Murchison Widefield Array (GLEAM; Hurley-Walker et al. 2017), and the 120-168 MHz LOFAR Twometre Sky Survey (LoTSS; Shimwell et al. 2017, 2019).

In the past 20 years, large surveys have also been carried out at optical wavelengths. These surveys include the Sloan Digital Sky Survey (SDSS; York et al. 2000), the $2^{\circ}$ Field Galaxy Redshift Survey (2dFGRS; Colless et al. 2001), the 2MASS Redshift Survey (2MRS; Huchra et al. 2012), the $6^{\circ}$ Field Galaxy Survey (6dFGS; Jones et al. 2009), and most recently the deep photometric survey called Panoramic Survey Telescope and Rapid Response System (Pan-STARRS; Kaiser et al. 2002, 2010; Chambers et al. 2016). The data from these surveys has allowed for the identification of many new GRGs, as shown in Dabhade et al. (2017).

High sensitivity to low surface brightness features and high spatial resolution to decipher the morphologies are key require- ments in identifying GRGs. LoTSS provides a combination of both these properties for the first time and hence by combining it with the SDSS/Pan-STARRS optical surveys, we used it to search for new GRGs in order to form a statistically significant sample. Our study of GRGs will be presented in two papers, where Paper I (this paper) reports on the methodology used for the systematic search scheme implemented for the discovery of new GRGs and GRQs from the LoTSS and presents the sample's radio properties. The Paper II will focus on the study of the host AGN and galaxy properties of the GRGs and GRQs sample and compare them with another sample (also from LoTSS) of NRGs matched in redshift as well as optical and radio luminosity to the GRG sample.

Throughout the paper, we adopt the flat $\Lambda \mathrm{CDM}$ cosmological model based on the latest Planck results $\left(H_{0}=67.8 \mathrm{~km} \mathrm{~s}^{-1} \mathrm{Mpc}^{-1}\right.$, $\left.\Omega_{\mathrm{m}}=0.308\right)$ (Planck Collaboration XIII 2016), which give a scale of $4.6 \mathrm{kpc} /{ }^{\prime \prime}$ for the redshift of 0.3 . In this paper, RGs with projected linear sizes $\geq 0.7 \mathrm{Mpc}$, which were computed using the above mentioned cosmological parameters are called GRGs. All images are in the $\mathrm{J} 2000$ coordinate system. We use the convention $S_{v} \propto v^{-\alpha}$, where $S_{v}$ is flux at frequency $v$ and $\alpha$ is the spectral index.

\section{Identifying new GRGs in LoTSS}

\subsection{The LoTSS first data release}

LoTSS is a $120-168 \mathrm{MHz}$ survey, which is being conducted with the high-band antennas (HBA) of LOFAR, will eventually cover the whole northern sky. Hardcastle et al. (2016) have already demonstrated the potential of LoTSS deep observations for discovering GRGs and found seven in the Herschel ATLAS North Galactic Pole survey area $\left(142 \mathrm{deg}^{2}\right)$. Here, we focus on the LoTSS first data release (LoTSS DR1; Shimwell et al. 2019). The LoTSS DR1 spans (J2000.0 epoch) right ascension from $10 \mathrm{~h} 45 \mathrm{~m}$ to $15 \mathrm{~h} 30 \mathrm{~m}$ and declination from $45^{\circ} 00^{\prime}$ to $57^{\circ} 00^{\prime}$ (HETDEX:Hobby-Eberly Telescope Dark Energy Experiment Spring field region), covering an area of $424 \mathrm{deg}^{2}$ with a median noise level across the mosaic of $71 \mu \mathrm{Jy}^{\text {beam }}{ }^{-1}$ and $\sim 6^{\prime \prime}$ resolution. In Fig. 1, we see a comparison between the LoTSS and other radio surveys, such as NVSS and FIRST, for a GRG from our sample. The top image (a) is an optical-radio overlay with a blue colour indicating LoTSS low frequency 6 " resolution map on the optical SDSS tri-colour image. In the bottom image (b), the same source is shown as it is observed in NVSS and FIRST. NVSS, though highly sensitive to large-scale diffuse emission, fails to reveal the finer details across the source and cannot properly resolve the core due to its coarser resolution of $45^{\prime \prime}$. The FIRST survey on the other hand has high resolution, which manages to resolve the core and hence helps in identifying the host AGN and galaxy but misses out on almost all the diffuse emission of the lobes and hence it alone cannot be used to identify RGs. The LoTSS data provides with both high resolution as well as high sensitivity and does not resolve out structures revealing finer details of the emission of large-scale jets. Both images (Fig. 1 and source 7 of Fig. A.1) clearly show the radio core and jets feeding the giant radio lobes and the hotspots. This clearly illustrates the excellence of LoTSS and its great potential in unveiling interesting sources.

By using the LoTSS DR1 radio data and optical-infrared data, a Value Added Catalogue ${ }^{1}$ (VAC) of 318520 radio sources has been created (Williams et al. 2019; DR1-II). The host

\footnotetext{
https://www . lofar-surveys.org/
} 


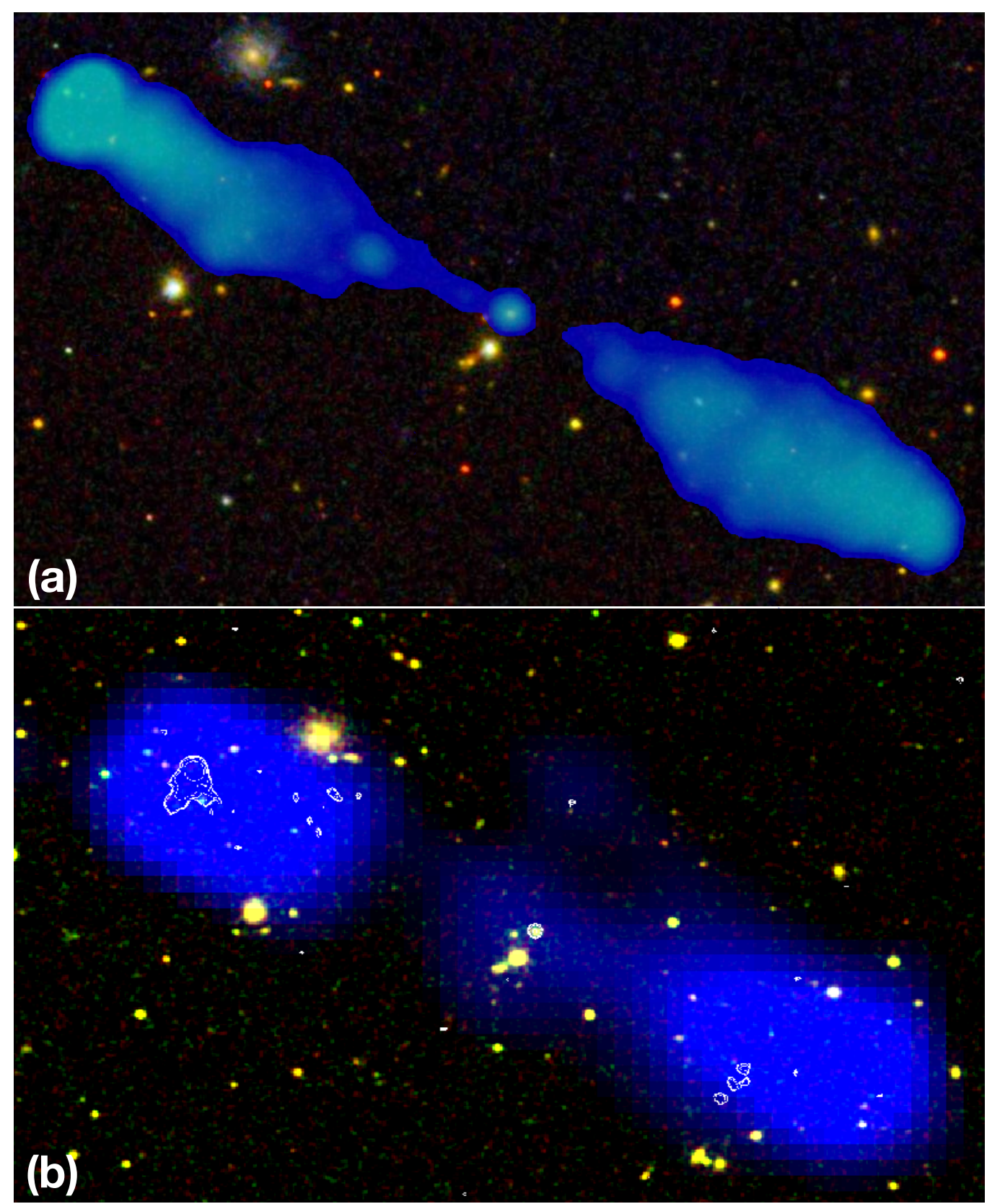

Fig. 1. Panel a: colour composite image of $1.86 \mathrm{Mpc}$ long GRG J105817.90+514017.70 made using LoTSS-DR1 144 MHz radio and optical SDSS image. Panel $b$ : colour composite image of the same object with optical and radio overlay, where blue represents $1400 \mathrm{MHz}$ NVSS image with $45^{\prime \prime}$ resolution and white contours from the $1400 \mathrm{MHz}$ FIRST survey having 5" resolution superimposed on optical SDSS image. The LoTSS $144 \mathrm{MHz}$ $6^{\prime \prime}$ resolution image shown in top panel clearly resolves the core and jet and also highlights the diffuse parts of the lobes, which is missed by the FIRST and unresolved in NVSS.

galaxies and quasars were identified using the Pan-STARRS and Wide-field Infrared Survey Explorer (WISE; Wright et al. 2010). The Pan-STARRS-AllWISE catalogue was cross matched with the LoTSS survey using a likelihood ratio method. Furthermore, human visual inspection was used for the final classification of complex radio sources employing the LOFAR Galaxy Zoo (LGZ), the details of which are given in Williams et al. (2019; DR1-II). The photometric redshift and rest-frame colour estimates for all hosts, including galaxies and quasars, of the matched radio sources are presented in Duncan et al. (2019; DR1-III). The VAC lists the radio properties, identification methods, and optical properties when available.

\subsection{Semi-automated search for GRGs}

The methodology of identifying GRGs and forming the final catalogue is presented in a flow chart as seen in Fig. 2 and is described below:

1. The VAC of 318520 radio sources was at first refined based on the point source completeness, which is $90 \%$ at an integrated flux density of $0.5 \mathrm{mJy}$ for LoTSS DR1 (Shimwell et al. 2019). We applied a flux density cut at this level. This results in the total number of sources reducing to 239845 .

2. Only objects with optical identification and redshift estimates were selected, resulting in 162249 sources. 


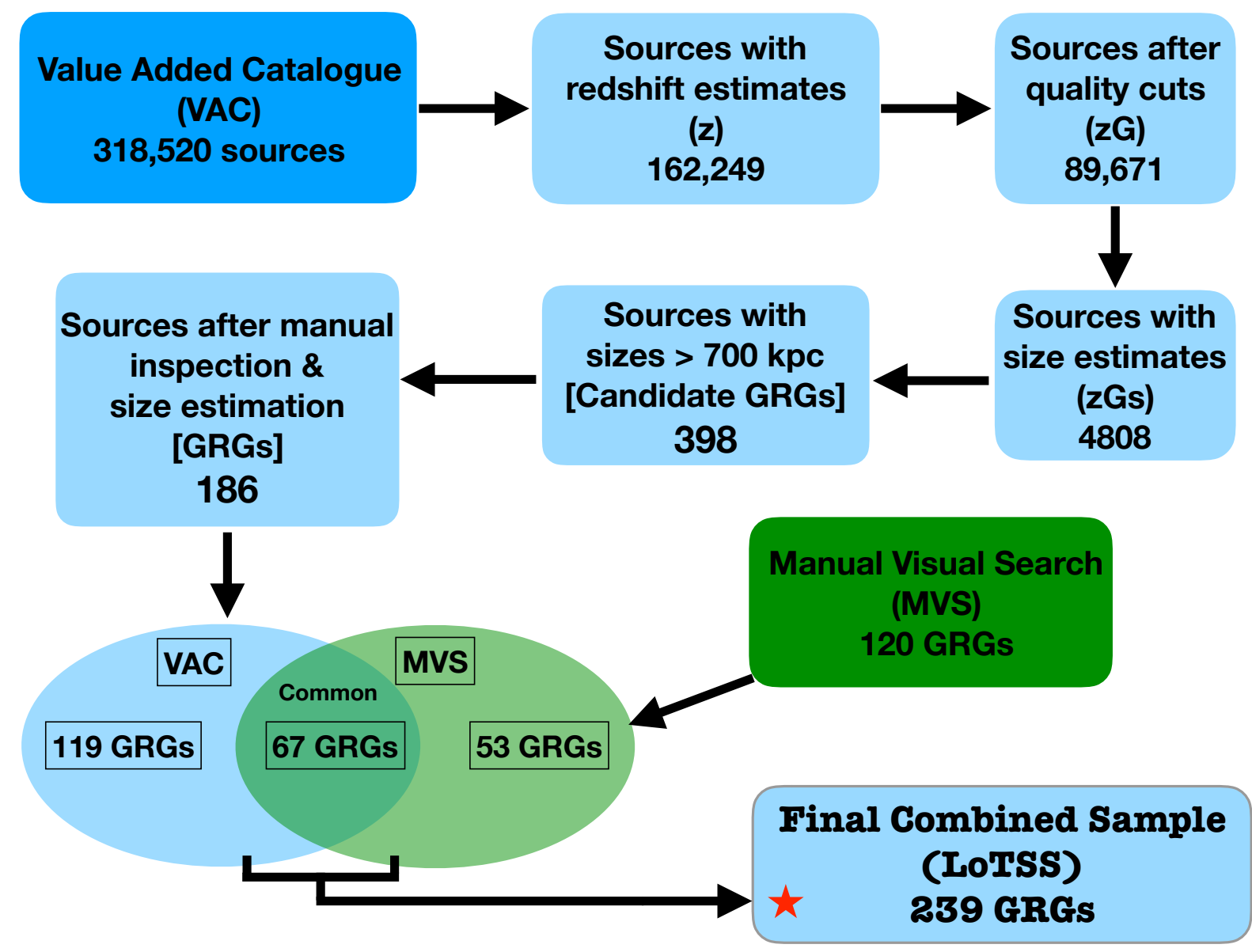

Fig. 2. Above figure shows schematics for finding GRGs from LoTSS DR1, using VAC and MVS in steps. More details are presented in Sect. 2.

3. The redshift information in the VAC was compiled by mainly using the SDSS spectroscopic data. For the sources that do not have spectroscopic redshift information from the SDSS or any other spectroscopic survey, Duncan et al. (2019) have estimated photometric redshifts by using multi-band photometry. For our work, we imposed a further photometric quality cut on the estimated photometric redshifts. Only sources satisfying the condition $\Delta z /(1+z)<0.1$ were selected, where $\Delta z$ is the halfwidth of the $80 \%$ credible interval. This results in a reduction of the sample from 162249 sources to 89671 sources.

4. Williams et al. (2019) inspected all the extended complex radio sources visually via the LGZ programme and estimated the angular size for them. A semi-automated way was adopted for this work by Williams et al. (2019). This resulted in an angular size estimation of a total of 13222 sources in the VAC.

We find that, of the 89671 sources with reliable redshifts, only 4808 sources have angular size estimates by the above mentioned method (LGZ). Or, in other words, 4808 sources have angular size estimates and have passed the photometric quality cut as well (point 3 ).

5. For all of the 4808 sources, we computed the projected linear size $(\mathrm{kpc})$ and only those that had an extension above $700 \mathrm{kpc}$ were considered for further analysis. This resulted in a sample of 398 candidate GRGs.

6. Further, the candidate GRGs were visually inspected by us in order to identify and remove those with uncertainty in the host, large asymmetry or a high degree of bending, or narrow angle tailed morphology. The angular size measurement was refined by taking the distance between the farthest points of the $3 \sigma$ contours of the source and the projected linear size was recom- puted. We measured the sizes of the sources by using the LoTSS DR1- low-resolution maps $\left(20^{\prime \prime}\right)$ as it shows the entire structure of the GRGs and also does not miss any diffuse emission. The final sample size of GRGs from the VAC is 186 GRGs.

\subsection{Manual visual search from LoTSS DR1}

An independent manual visual search (MVS) was also carried out to search for GRGs in LoTSS DR1 to look for additional GRGs that were missed in the semi-automated method employed on the VAC. In this approach, all mosaics from LoTSS DR1 were scanned for extended double-lobed structures (candidate GRGs). The radio core of the candidate GRGs were searched in the optical band (SDSS and PAN-STARRS) and infrared (IR) band (WISE) for counterparts (host galaxies). We also used other radio surveys, such as FIRST, TGSS, and WENSS, for additional information and consistency checks. This method (MVS) yielded 53 additional GRGs. These 53 GRGs were missed by the selection criteria because of the photometric redshift cuts we imposed or because the complex nature of the source structures lead them to not be fully characterised in the VAC.

\subsection{Final catalogue: VAC and MVS}

We combined both GRG samples (VAC and MVS) to form the final GRG catalogue of 239 GRGs (Table A.1). The final catalogue of 239 GRGs was cross matched with the GRG catalogue of Kuźmicz et al. (2018), which is a large compendium of GRGs published until 2018, along with other GRGs reported in the latest research papers. We find only 13 of our 239 GRGs were 
already known (listed in 13th column of Table A.1). The highresolution 6" LoTSS images at $144 \mathrm{MHz}$ of GRGs can be found in Figs. A.1-A.8, where the white contours represent emission seen in the LoTSS low-resolution $20^{\prime \prime}$ map at $144 \mathrm{MHz}$. The position of the host galaxy is marked with a white cross " + ".

\section{Results: The LoTSS catalogue of GRGs}

Our search of LoTSS DR1 has enabled us to construct a cata$\operatorname{logue}^{2}$ of 239 GRGs. With the high sensitivity of LoTSS, we are able to detect GRGs as faint as $\sim 2.5 \mathrm{mJy}$ in total flux at $144 \mathrm{MHz}$. Using the available optical data and radio data, we have computed the radio powers, spectral indices, and classified the morphological types for the sample of 239 GRGs (Table A.1). The GRGs from our sample have sizes in the range of $0.7 \mathrm{Mpc}-\sim 3.5 \mathrm{Mpc}$ (Fig. 3) with a median size of $0.89 \mathrm{Mpc}$ and a mean size of $1.02 \mathrm{Mpc}$. GRGs with sizes greater than $2 \mathrm{Mpc}$ are very rare within the GRG population and in our sample we find 7 out of the 239 GRGs to have sizes $\geq 2 \mathrm{Mpc}$.

\subsection{Optical host properties}

The GRGs in our sample span a wide redshift range from 0.1 to 2.3 (Fig. 4). GRGs that are hosted by galaxies are not selected beyond $z \sim 1$ due to sensitivity limits of the optical surveys. We used the SDSS DR14 Quasar catalogue (Pâris et al. 2018) and SDSS to identify the GRGs hosted by quasars in our sample. We note that 151 out of 239 GRGs have optical spectroscopic redshifts from SDSS. Of the 151 GRGs with optical spectroscopic redshifts 40 are hosted by quasars (GRQs). Interestingly, based on the available optical data (SDSS and Pan-STARRS) and the Galaxy Zoo catalogue of spiral galaxies (Hart et al. 2016), we find that none of the GRGs is hosted by a spiral galaxy.

Most of the redshifts of the hosts of GRGs were obtained from the VAC, which has made use of the spectroscopic data from the SDSS and has estimated photometric redshifts for the sources that do not have spectroscopic data in SDSS or in other literature. The uncertainties in the photometric redshifts are explained in Sect. 3 (especially Sect. 3.5) of Duncan et al. (2019).

\subsection{Spectral index $\left(\alpha_{144}^{1400}\right)$}

The LoTSS DR1 provides maps of the radio sky (centred at $144 \mathrm{MHz})$ at two resolutions, high $\left(6^{\prime \prime}\right)$ and low $\left(20^{\prime \prime}\right)$, which enables us to identify compact cores, jets, and hotspots as well as see the extent of diffuse emission. To compute the flux at $144 \mathrm{MHz}$, we used the 20" low-resolution maps of LoTSS DR 1 (Col. 8 of Table A.1). Measurement in the flux errors is done by taking a $20 \%$ calibration error for LoTSS DR1 (Shimwell et al. 2019). By combining high frequency (1400 MHz) NVSS and low frequency (144 MHz) LoTSS, we computed the integrated spectral index $\left(\alpha_{144}^{1400}\right)$ for GRGs in our sample (Col. 10 of Table A.1). The NVSS radio map cutouts for GRGs were obtained from its server $^{3}$ from which we measured the flux of the source.

The following steps were adopted to obtain the spectral index:

\footnotetext{
2 Before the precise measurement of $H_{0}$, originally GRGs were defined as RGs with projected linear sizes $\geq 1 \mathrm{Mpc}$ using $H_{0}=50 \mathrm{~km} \mathrm{~s}^{-1} \mathrm{Mpc}^{-1}$. If we convert the original definition with the latest precise measurement of $H_{0}=67.8 \mathrm{~km} \mathrm{~s}^{-1} \mathrm{Mpc}^{-1}$ with $\Omega_{\mathrm{m}}=0.308$, then the lower limit of the projected linear size of GRGs is $\sim 0.74 \mathrm{Mpc}$. In our sample, 28 GRGs have projected linear sizes between $0.7 \mathrm{Mpc}$ and $0.74 \mathrm{Mpc}$.

3 https://www.cv.nrao.edu/nvss/postage.shtml
}

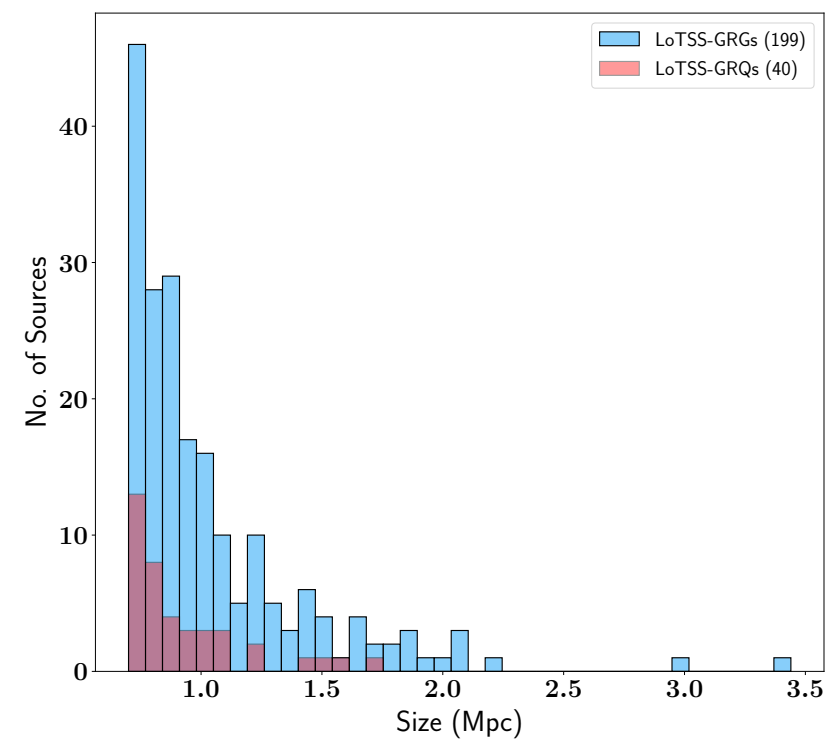

Fig. 3. Histogram for sizes of GRGs and GRQs from the LoTSS sample.

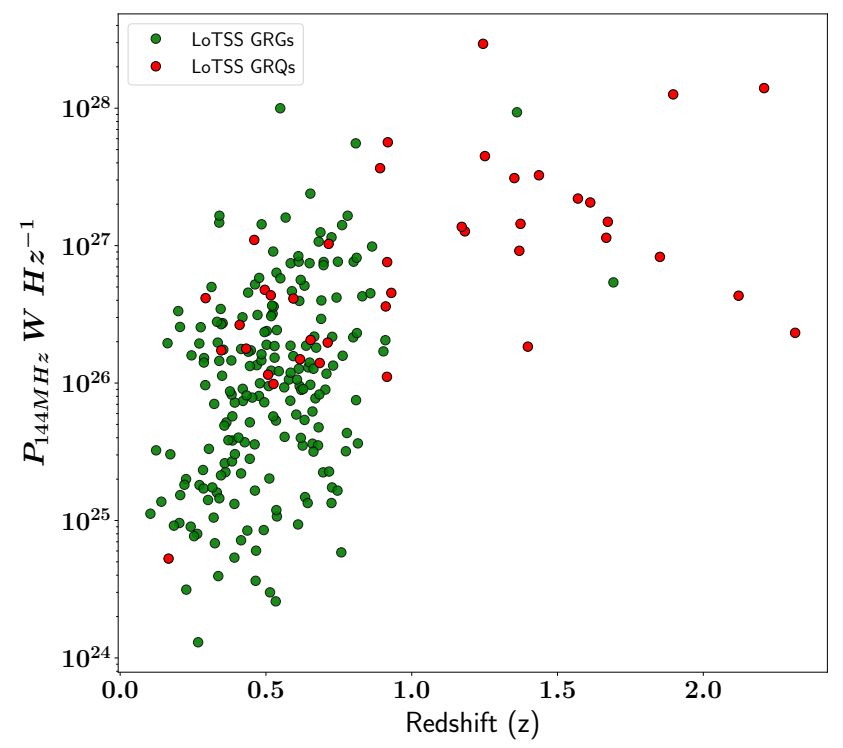

Fig. 4. Radio power and redshift distribution for GRGs and GRQs from LoTSS. The two galaxies marked in a green colour with $z \geq 1$ are quasar candidates as explaind in Sect. 3.4.

- We convolved the LoTSS low-resolution images (cutouts of GRGs from main mosaics) to the same resolution of NVSS $\left(45^{\prime \prime}\right)$ and regridded the LoTSS images to match with NVSS.

- We made automated masks (regions to extract flux) using the package PyBDSF ${ }^{4}$ (Python Blob Detection and Source Finder) of Mohan \& Rafferty (2015).

- The 45" convolved LoTSS maps were manually inspected for possible contamination (using the high-resolution LoTSS maps ( $\left.6^{\prime \prime}\right)$ and FIRST's 5" maps) from other sources in the field and manual masks were made. The flux was obtained by considering only the region in the manual masks from the automated masks.

- Finally, by using the fluxes obtained from NVSS and LoTSS, the integrated spectral index was computed for the whole source.

A total of 37 out of the 239 sources were contaminated by other nearby radio sources in the low-resolution convolved

\footnotetext{
4 http://www.astron.nl/citt/pybdsf/
} 


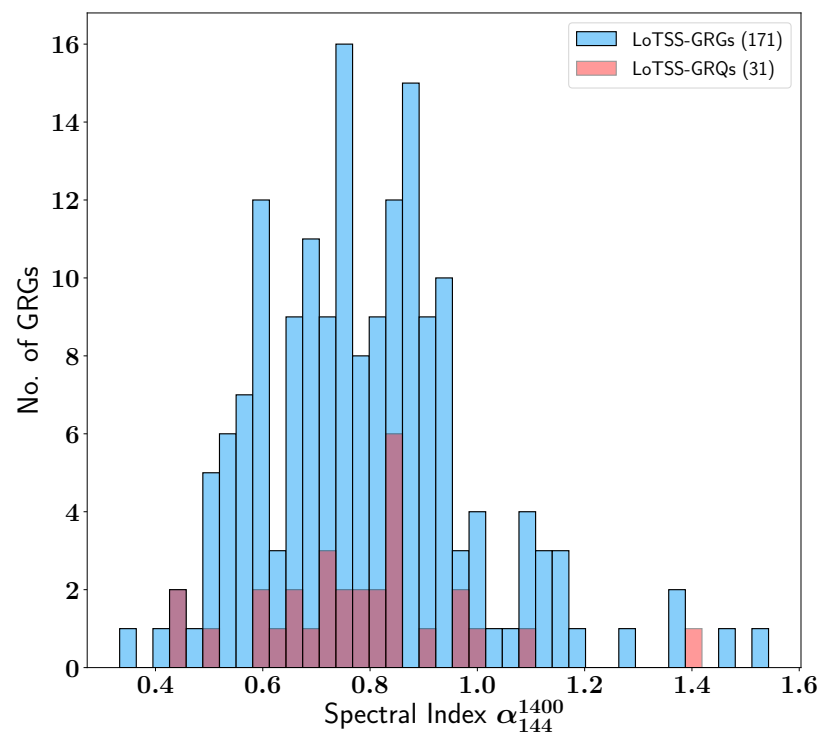

Fig. 5. Histogram of Spectral index $\left(\alpha_{144}^{1400}\right)$ of GRGs and GRQs in LoTSS DR1 sample made using LoTSS and NVSS.

LoTSS maps and NVSS maps, for these sources (marked with "-" in Col. 10 of Table A.1) a spectral index was not computed. For sources with no detection in NVSS, upper limits on the flux were computed, and a spectral index limit was obtained (indicated with $<$ sign in Col. 10 of Table A.1).

Figure 5 shows the spectral index $\left(\alpha_{144}^{1400}\right)$ distribution of 171 GRGs and 31 GRQs. The median and mean values for spectral index of GRGs are 0.77 and 0.79 , respectively. Similarly, for GRQs, the median and mean values are 0.78 and 0.76 , respectively. These mean spectral index values of GRGs and GRQs are similar to those of NRGs (Oort et al. 1988; Gruppioni et al. 1997; Kapahi et al. 1998; Ishwara-Chandra et al. 2010; Mahony et al. 2016). In the work of Oort et al. (1988), they surveyed the Lynx field with the Westerbork Synthesis Radio Telescope (WSRT) at $325 \mathrm{MHz}$ and $1400 \mathrm{MHz}$. Gruppioni et al. (1997) surveyed the Marano field using the Australia Telescope Compact Array (ATCA) at $1.4 \mathrm{GHz}$ and $2.4 \mathrm{GHz}$. Kapahi et al. (1998) surveyed the Molonglo Radio Catalogue sources with the VLA at $L$ and $S$ bands. Ishwara-Chandra et al. (2010) surveyed the LBDS-Lynx field (LBDS: Leiden-Berkeley Deep Survey) using the $150 \mathrm{MHz}$ band of the Giant Metrewave Radio Telescope and other archival data from GMRT at $610 \mathrm{MHz}$ and $325 \mathrm{MHz}$ along with data from other surveys, such as WENSS, FIRST, and NVSS. Mahony et al. (2016) surveyed the Lockman Hole field using LOFAR $150 \mathrm{MHz}$ and WSRT $1.4 \mathrm{GHz}$. All the above work obtained spectral index values in the range of $\sim 0.7-0.8$ and therefore the 0.75 value is often assumed as the average spectral index value for RGs in the absence of any multi-frequency observations.

The above mentioned result implies that RGs and GRGs do not differ in terms of their spectral index properties, and most of the GRG population are active RGs and not dead or remnant RGs. The values for the spectral index of GRGs provided by us are integrated values for the whole source and are not just one component of GRGs (such as a core or lobes). It has been found that for some GRGs, the lobes come out to be steeper than usual, which is obtained by multi-frequency (observations at 3 to 4 radio bands) observations of individual sources. There are examples (source number 16, 17, and 193) in our sample where the sources are core dominated (maximum flux observed in radio core) and this, therefore, influences sources to have flatter (less than 0.5 ) overall spectral index.

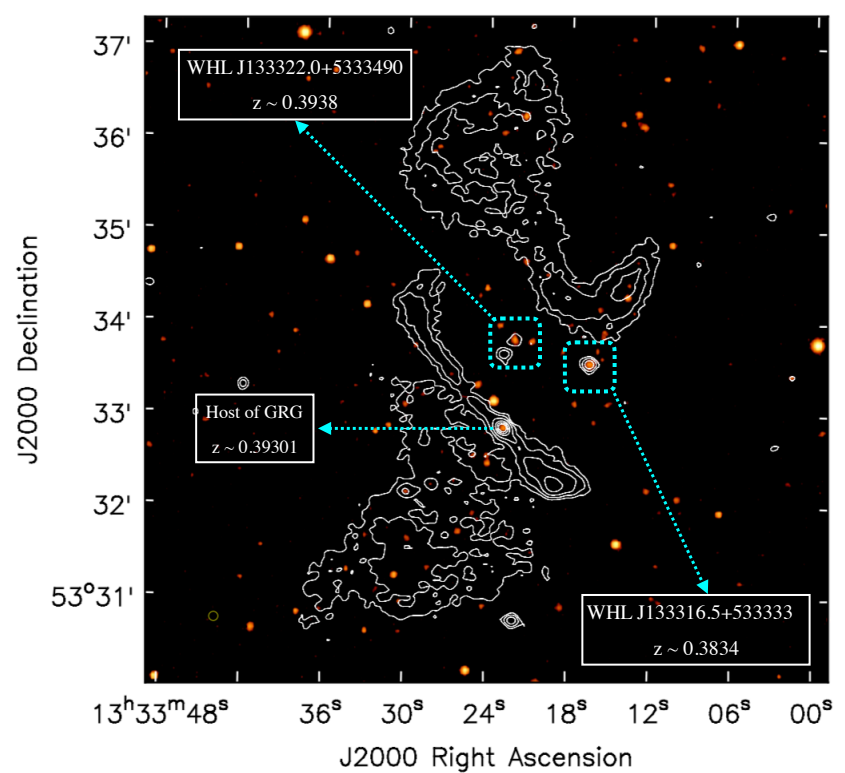

Fig. 6. GRGJ133322.79+533250.94 possibly amidst galaxy cluster relics. The background image in an orange colour is SDSS I band optical image, which is superimposed with LoTSS DR1 high-resolution $\left(6^{\prime \prime}\right)$ contours.

\subsection{Notes on individual objects}

Below, we present brief notes on a selection of some of the most interesting objects in our sample.

\subsubsection{Source 5 - GRG J105725.96+492900.31}

The host galaxy does not perfectly coincide with the bright corelike feature as they are separated by $\sim 5^{\prime \prime}$ distance from one another. It is most likely a knot in the jet, which is located right next to a faint radio core. There is neither a galaxy in the immediate vicinity nor any other alternate compact radio emission along the axis of the source, which could possibly be an alternate host of this source.

\subsubsection{Source 10 - GRG J110433.11+464225.76}

This unusual source has a well detected radio core but does not exhibit any jets or well formed lobes. It has diffuse emission on either side of the radio core and has been reported by Thwala et al. (2019) to be a relic RG with a size of $0.86 \mathrm{Mpc}$, thereby making it a GRG. Towards the north-western side of the source, there exists an independent FR-II type RG. Using the higher -resolution maps the independent source's flux was measured and then subtracted from the LoTSS's low-resolution maps in order to avoid contamination of source fluxes.

\subsubsection{Source 44 - GRG J113931.77+472124.3}

As seen in Fig. A.2, there is a compact radio source north of the "+" marker (host galaxy-radio core), which is an independent source. Also, the bright compact source seen towards the southeastern side is independent of the GRG J113931.77+472124.3.

\subsubsection{Source 61 - GRG J121555.53+512416.41 and Source 162 - GRG J135628.50+524219.23}

These sources are core dominated objects at both low and high frequencies. The lobes of both sources are not detected in NVSS. These are possibly candidates of revived and rejuvenated GRGs as we only observe the diffuse lobes at low frequencies (LoTSS), 


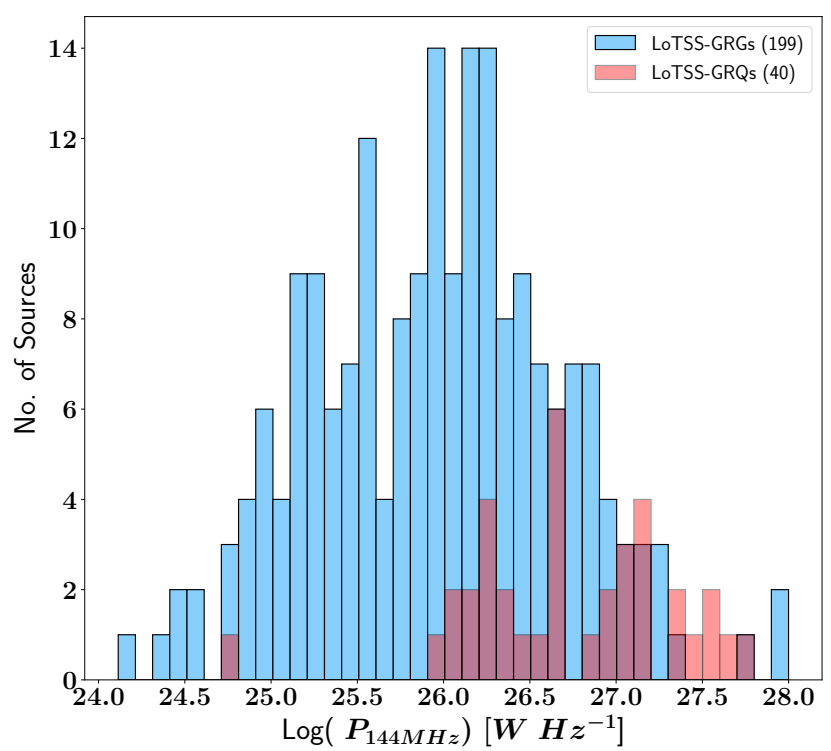

Fig. 7. Histogram of radio power at $144 \mathrm{MHz}$ of GRGs and GRQs.

which could be from the previous epoch. The presence of the bright core may indicate restarting activity.

\subsubsection{Source 136 - GRG J133322.79+533250.94}

This source displays a peculiar morphology and possibly resides in a unique environment. The VAC estimates its photometric redshift $(z)$ to be $0.3539 \pm 0.0344$. Lopes (2007) estimated its photometric redshift based on SDSS data to be $0.39301 \pm 0.02578$. GRGJ133322.79+533250.94 is close to two galaxy clusters, namely WHL J133322.0+5333490 at redshift of 0.3938 and WHL J133316.5+533333 at a redshift of 0.3834. Based on the photometric redshift of Lopes (2007), GRG J133322.79+533250.94 is plausibly to be associated with the galaxy cluster WHL J133322.0+5333490 as they are at similar redshifts and are separated by $\sim 1^{\prime}$. This GRG appears to reside in an environment of a possible merger of two galaxy clusters and the associated radio relic can be seen north of the GRG (Fig. 6).

\subsubsection{Source 161 - GRG J135414.72+491315.2}

This GRG exhibits typical FR-II morphology. Interestingly, the radio core coinciding with a faint galaxy is only detected in the FIRST survey.

\subsubsection{Source 221 - GRG J145002.36+540528.27}

The radio core is well detected in the FIRST survey, which coincides with a galaxy. The source is sufficiently resolved only in the LoTSS high-resolution map as it is located right next to a bright source. The spectral index was computed by estimating an upper limit for the flux from NVSS.

\subsubsection{Source 222 - GRG J145057.28+530007.76}

The radio core is only resolved in the FIRST survey. It is not symmetrically placed between the two lobes and it is closer to the eastern lobe.

\subsubsection{Source 237 - GRG J151835.37+510410.70}

The radio core is not symmetrically placed between the two lobes and is closer to the northern lobe. It is also well detected in the FIRST survey. The southern lobe shows a prominent hotspot.

\subsection{Giant radio quasars}

GRGs with quasars as their AGN (GRQs) are even rarer than the GRGs with non-quasar AGN. Based on the catalogue of Kuźmicz et al. (2018) and the candidates presented by Kuligowska \& Kuźmicz (2018), there are currently only $\sim 70$ GRQs known and less than $\sim 10$ GRQs known at $z \geq 2$. From our LoTSS sample of 239 GRGs, 40 are confirmed GRQs and two are candidate GRQs $\left(Q_{\mathrm{c}}\right)$. A total of only six GRQs of the 40 confirmed GRQs were previously reported (Col. 13 in Table A.1) and the rest are all new findings. Our sample of $\sim 40$ GRQs significantly increases the sample of the total GRQs known, at present, from 70 (Kuźmicz et al. 2018) to more than 100. The GRQs in our sample have median and mean radio powers of $6.2 \times 10^{26} \mathrm{~W} \mathrm{~Hz}^{-1}$ and $2.4 \times 10^{27} \mathrm{~W} \mathrm{~Hz}^{-1}$ at $144 \mathrm{MHz}$, respectively, as seen in the histogram distribution of radio powers (Fig. 7).

GRQs J110833.99+483203.03, J123933.21+500708.01, and $\mathrm{J} 133418.63+481317.08$ are the three GRQs that we found at a very high redshift of $z \geq 2$. They are shown in Fig. 8 . GRG J110833.99+483203.03 (16 in Table A.1) is a highly core dominated object at both high as well as low frequencies and hence exhibits a very flat spectral index of $\sim 0.2$. GRGs J132554.31+551936.23 and J141408.45+484156.11 (126 and 178 in Table A.1) have high redshifts and are the two quasar candidates $\left(Q_{\mathrm{c}}\right)$ as indicated by Richards et al. $(2009,2015)$. These objects have all the properties of a radio loud quasars but are still candidates because of the absence of an optical spectra. Therefore, they are not labelled as quasars and are not marked in a red colour in any of the figures.

\subsection{Environment analysis of GRGs}

We used two optically selected galaxy cluster catalogues to identify the host of GRGs with the brightest cluster galaxies (BCGs), which are found to be at the centres of galaxy clusters. We chose the following two catalogues as they are made using SDSS and have an overlap with the HETDEX region, which is common for LoTSS DR1.

Firstly, we used the galaxy cluster catalogue of Wen et al. (2012; hereafter WHL cluster catalogue), which consists of 132,684 clusters. They used photometric data from SDSS-III to find the clusters. This is the biggest galaxy cluster catalogue made using SDSS in the redshift range of $0.05<z<0.8$ and is $\sim 95 \%$ complete for clusters with a mass of $M_{200}>10^{14} M_{\odot}$ in the redshift range of $0.05<z<0.42$. A total of 71 GRGs from our sample fall in this redshift range.

We find 17 GRGs to be BCGs based on the WHL cluster catalogue. We also used the Gaussian Mixture Brightest Cluster Galaxy (GMBCG) catalogue (Hao et al. 2010) consisting of 55880 galaxy clusters, which is more than $90 \%$ complete within the redshift range of $0.1<z<0.55$. We find three extra GRGs to be BCGs from the GMBCG catalogue. Therefore, we find a total of 20 GRGs (Table A.2) from our sample of 239 to be BCGs residing in dense cluster environments and hosted by BCGs of the clusters. The mass and radius (obtained from Wen et al. 2012) of the 17 clusters are listed in Table A.2.

\section{Discussion}

\subsection{GRGs with sizes $>2 M p c$}

GRGs with projected linear sizes $>2 \mathrm{Mpc}$ are quite rare and as observed from Fig. 3, there are only 7 GRGs with sizes greater than $2 \mathrm{Mpc}$ in our sample of 239. Based on a large but incomplete sample compilation of all the currently known GRGs of $\sim 400$ sources, 

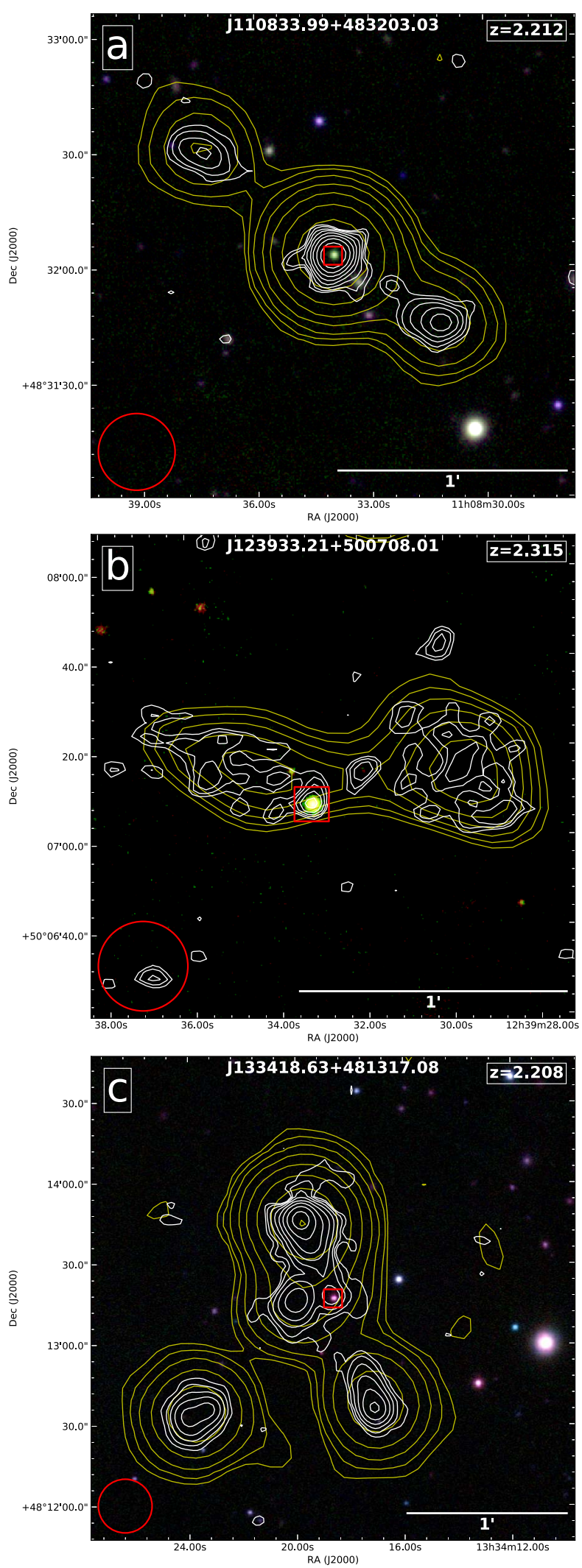

Fig. 8. Optical-radio overlay of high redshift $(z>2)$ GRGs, where the optical images colour composite of three bands of PanSTARRS and radio is from the LoTSS DR-1 at two resolutions. The red square indicates the host of the three GRQs. The white and yellow colour contours represent LoTSS high $\left(6^{\prime \prime} \times 6^{\prime \prime}\right)$ and low $\left(20^{\prime \prime} \times 20^{\prime \prime}\right.$; red circles at bottom left) resolution maps, respectively, which have eight equally spaced relative contour levels starting from three times $\sigma$, where $\sigma$ denotes the local rms noise. only $\sim 62$ GRGs $(\sim 15 \%)$ have sizes greater than $2 \mathrm{Mpc}$ (Willis et al. 1974; Laing et al. 1983; Lacy et al. 1993; Ishwara-Chandra \& Saikia 1999; Schoenmakers et al. 2001; Machalski et al. 2001, 2007; Lara et al. 2001; Saripalli et al. 2005; Kozieł-Wierzbowska \& Stasińska 2011; Solovyov \& Verkhodanov 2014; Amirkhanyan et al. 2015; Amirkhanyan 2016; Dabhade et al. 2017; Clarke et al. 2017; Prescott et al. 2018; Sebastian et al. 2018).

If we take a complete radio sample, such as 3CRR (Laing et al. 1983), then we have only 12 GRGs from the total sample of 173 , which is only $\sim 7 \%$ of the total population. Considering this sample of 12 GRGs from $3 \mathrm{CRR}^{5}$, there are only two sources with sizes greater than $2 \mathrm{Mpc}$, which constitutes $\sim 17 \%$ of the total GRG population in the 3CRR complete sample. The conditions under which a RG grows in order to become a GRG is not very well understood and studies so far based on the observations and theory seem to suggest that the gigantic size could be due to an interplay between the jet power and the environment.

\subsection{Morphology of GRGs}

Most known GRGs exhibit FR-II-type morphology and we very rarely see RGs with Mpc-scale size with FR-I-type morphology. Based on their morphologies, we classify 18 GRGs as FR-I type and 215 GRGs as FR-II type. GRGJ121900.76+505254.41 is the only FR-I GRG residing at cluster centre and hosted by a BCG (Table A.2).

The total radio power of FR-I sources on average is less than that of the FR-II sources (Ledlow \& Owen 1996; Lara et al. 2004). As seen in Fig. 9, the FR-I type of GRGs have a limited range in radio power $10^{24} \sim 10^{26} \mathrm{~W} \mathrm{~Hz}^{-1}$, whereas the FR-II exhibits a wide range of radio powers from $10^{24} \sim 10^{28} \mathrm{~W} \mathrm{~Hz}^{-1}$ at $144 \mathrm{MHz}$.

\subsubsection{GRGs with hybrid morphology}

Radio galaxies that show FR-I morphology on one side and FRII morphology on the other side are referred to as HyMoRS (Gopal-Krishna \& Wiita 2002). The earliest example of this morphology was presented and studied by Saikia et al. (1996), for the RG 4C +63.07 . They attributed this to an intrinsic asymmetry in either the collimation of its jets or the supply of fuel from the central black hole to the opposite sides.

Gawroński et al. (2006) estimated the occurrence of HyMoRS to be as low as $\leq 1 \%$ amongst the RG population. Recently, Kapińska et al. (2017) presented 25 new candidate HyMoRS, of which five are GRGs with one being at the centre of a galaxy cluster. Therefore, one of the possible scenarios for such hybrid morphology could be attributed to different environments on each side of the host galaxy (radio core).

In our sample of 239 GRGs, we find six examples of HyMoRS (identified via visual inspection), which are listed in Col. 11 (FR type) of Table A.1 and indicated with the roman numeral III, images of these sources are presented in Fig. 10. This is by far the largest HyMoRS GRG sample to have ever been reported. Environment, as well as host AGN studies, are needed to understand this class of RGs, which can also grow to megaparsec scales in size.

\subsubsection{DDRGs: Double double radio galaxies}

DDRG are FR-II-type objects with two pairs of lobes, which is indicative of their restarted nature (Schoenmakers et al. 2000; Saikia \& Jamrozy 2009). The newly created jets in such sources

\footnotetext{
http://www.jb.man.ac.uk/atlas/
} 


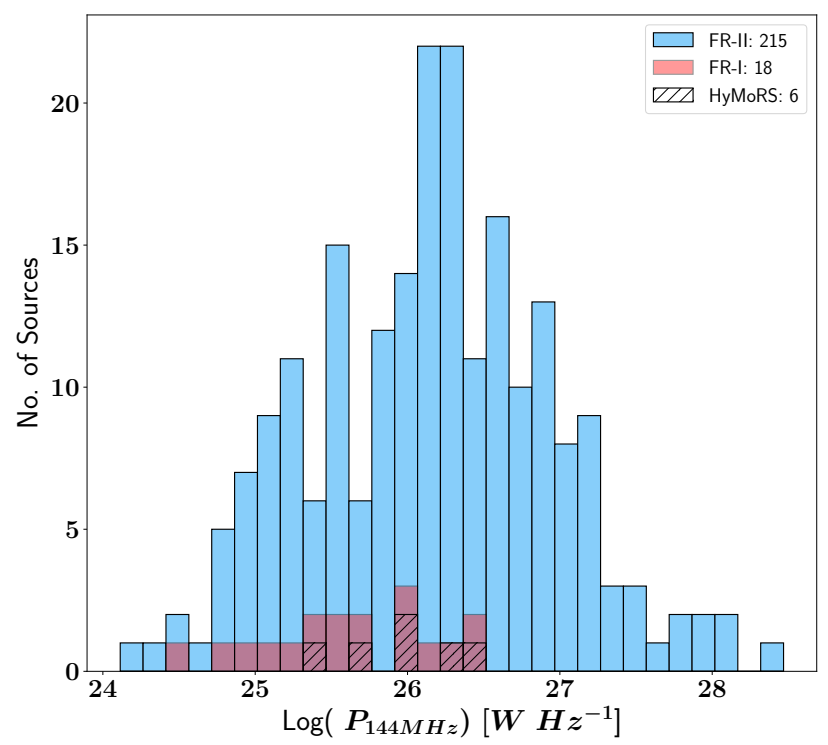

Fig. 9. Radio power $(144 \mathrm{MHz})$ distribution of FR-I, FR-II, and HyMoRS souces in LoTSS DR1 sample of giants.

travel outwards through the cocoon formed by the earlier cycle and episode of activity rather than the common intergalactic or intracluster medium, after moving through the interstellar medium of the host galaxy. In general, the outer double lobes are aligned with the inner ones and can extend from a few kpc up to Mpc scales. It is likely that in DDRGs, due to an unknown mechanism or activity, the interruption of these bipolar relativistic jet flows has occurred leading to such morphologies.

In the last approximately 20 years only about 140 DDRGs have been reported (Schoenmakers et al. 2000; Nandi \& Saikia 2012; Kuźmicz et al. 2017; Mahatma et al. 2019; Nandi et al. 2019). Recently, (Mahatma et al. 2019) created a new sample of 33 DDRGs using the LoTSS DR1 and follow-up observations at higher frequency with the Jansky Very Large Array (JVLA), where they compared the optical and IR magnitudes and colours of their host galaxies with a sample of normal RGs. They find that the host galaxy properties of both DDRGs and normal radio galaxies are similar and suggest that the DDRG activity is a regular part of the life cycle of the RGs. We have found only 14 GRGs, which is currently the largest sample reported, in our entire sample with a DDRG-type morphology as seen in Figs. 11 and 12, indicating that megaparsec-scale DDRGs are rare. Except J110457.07+480913, $\mathrm{J} 110613.55+485748.29$, J121900.76+505254.41, J123754.05+ $512201.28, \mathrm{~J} 134313.31+560008.35, \quad$ and $\mathrm{J} 141504.70+$ 463428.97, the remaining eight were previously classified as DDRGs in the literature (seven in Mahatma et al. 2019 and GRG J140718.48+513204.63 in Nandi \& Saikia 2012).

We note that sources 28 (GRG JJ112130.35+494208.14) and 221 (GRGJ145002.36+540528.27) show some signs of being DDRGs but based on the current radio maps available to us, they cannot be confirmed as DDRGs and hence are candidate DDRGs. Deep and higher resolution observations at higher frequencies $(>1 \mathrm{GHz})$ are required to confirm the exact nature of candidate DDRGs.

These sources provide a unique opportunity to study the timescales of AGN recurrent activity. As seen in Figs. 11-13, some components of the giant DDRGs are very faint and it was only possible with LoTSS's high sensitivity and resolution to detect and sufficiently resolve them. Further studies on host AGN and galaxy properties of DDRGs along with their local environments are needed to understand these peculiar sources better.

\subsection{GRGs in dense environments}

It has been hypothesised that the growth of GRGs to enormous size is favoured by their location in low density environments. Using our large new sample of GRGs, it is now possible to test this hypothesis for objects with low redshifts $(z<0.55)$. We find that at least $8.4 \%$ of GRGs (Table A.2) from our sample (20 out of 239) are located in high density environments and are hosted by BCGs. This low number of BCG GRGs is possibly due to the absence of data for high redshift clusters in the WHL cluster catalogue and GMBCG catalogue, which are more sensitive to clusters with a redshift less than $\sim 0.42$ and $\sim 0.55$, respectively (see Sect. 3.5). We have 128 GRGs with $z \leq 0.55$, therefore at least $\sim 16 \%$ (20 out of 128 ) GRGs are in dense cluster environments. Based on the work of Paul et al. (2017), virialised structures of mass $M_{200} \geq 0.8 \times 10^{14} M_{\odot}$ are classified as a cluster of galaxies and non-virialised gravitionally bound structures consisting of few galaxies with $M_{200}<0.8 \times 10^{14} M_{\odot}$ are classified as a group of galaxies. By using this classification, there are 14 GRGs in clusters and 3 GRGs in groups of galaxies (see Table A.2).

Croston et al. (2019) carried out a study of environments of $\sim 8000$ radio loud AGNs from the LoTSS, where they find that only $10 \%$ of AGNs are associated with high density environments, similar to galaxy groups and clusters as well as AGNs with $L_{150}>10^{25} \mathrm{~W} \mathrm{~Hz}^{-1}$ (where $L_{150}$ is the radio power at $150 \mathrm{MHz}$ ), and are more likely to be in cluster environments. Similarly, our 20 BCG GRGs, which are also radio loud AGNs in galaxy cluster, exhibit $P_{144 \mathrm{MHz}}$ or $L_{150}>10^{25} \mathrm{~W} \mathrm{~Hz}^{-1}$.

To compare the properties of galaxy clusters that have radio loud BCGs (also BCG-GRGs) and the galaxy clusters with radio quiet BCGs, we extracted all the galaxy clusters present in the LoTSS-DR1 HETDEX region from the WHL cluster catalogue. This resulted in a total of 5027 galaxy clusters below redshift $(z)$ of 0.55 (highest redshift of BCG-GRG in our sample). Next, we cross-matched the location of BCGs (5027) from the WHL cluster with the VAC and FIRST catalogue to determine all the radio loud BCGs in this region. The final number of galaxy clusters with radio loud BCGs is 1559 (unique sample after combining the results from matches with VAC and FIRST). The 1559 radio loud BCGs also include our 17 BCG-GRGs (found in WHL; see Table A.2). In Fig. 14, we show the distribution of $M_{200}$ of clusters of galaxies, where $M_{200}$ is the mass of the cluster within $r_{200}$, and $r_{200}$ is the radius within which the mean density of a cluster is 200 times the critical density of the Universe. We note that about $30 \%$ (1541) clusters have radio loud BCGs that are not giants and only $\sim 0.34 \%$ of the clusters in WHL cluster catalogue (HETDEX region) host a GRG (or have BCG GRG). Though the GRG sample is less here but more or less complete, it seems that GRGs that are BCGs tend to avoid very high mass clusters and prefer less dense clusters.

The BCG GRGs can also possibly trace the inhomogeneities in the intergalactic gas, which could be one of the determining factors for the growth and evolution of these giant sources. The forward propagation of jets as well as the backflow from hotspots in the lobes of GRGs are influenced by the gas they encounter. Only 1 out of 20 BCG GRGs in our sample have FRI-type radio morphology and the remaining 19 have FR-II-type radio morphology. Almost all of these sources do not exhibit highly linear structures, but they are distorted or bent to a certain extent, which indicates the effect of interaction between the cluster medium and the expanding radio jets of the BCG GRGs. Remarkably, despite the possible resistance presented by the cluster medium, these sources have managed to grow to megaparsec scales. This seems to suggest the presence of an extraordinarily powerful AGN powering these sources. 

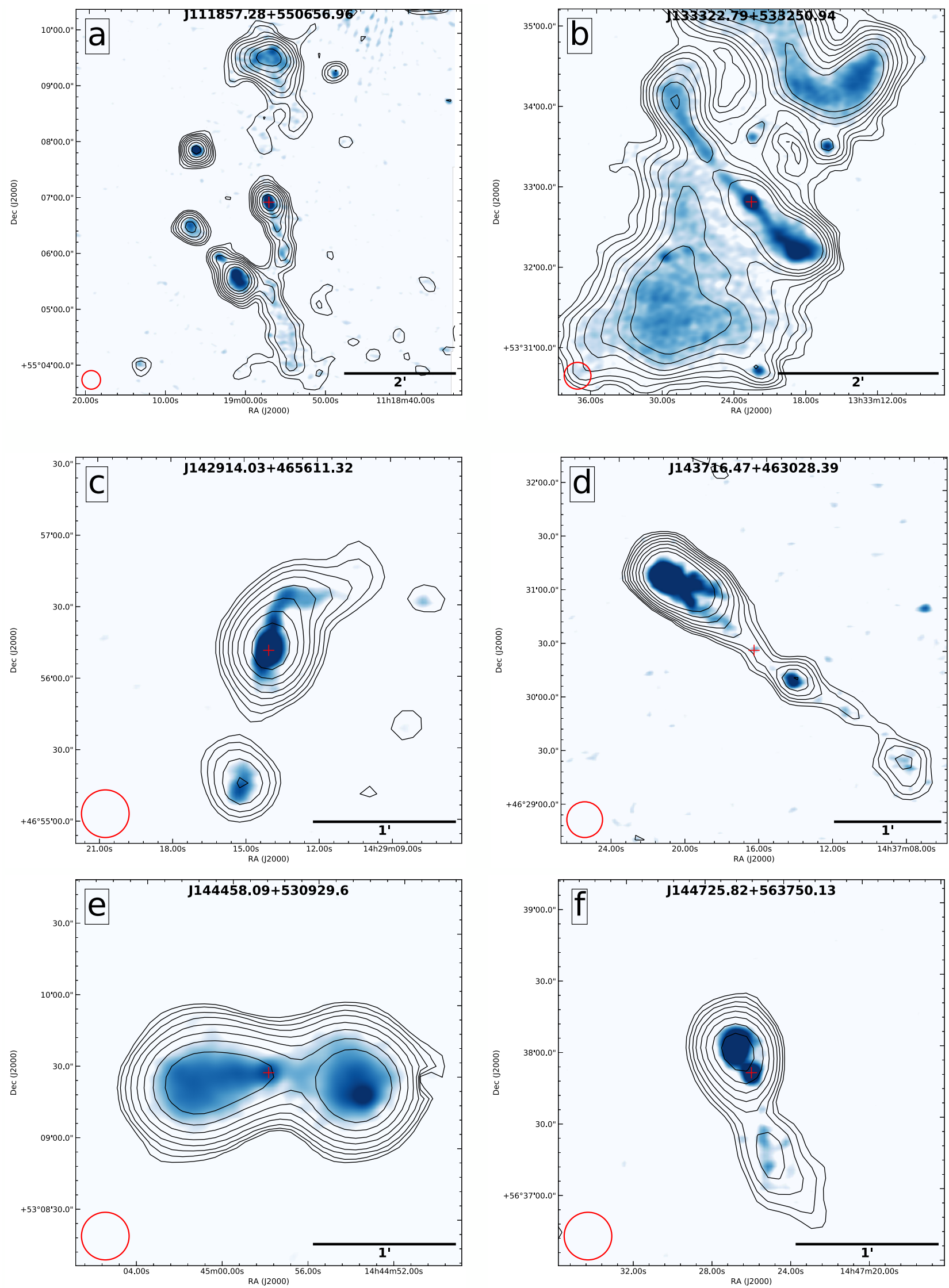

Fig. 10. LoTSS DR1 $\left(6^{\prime \prime} \times 6^{\prime \prime}\right)$ maps of 6 HyMoRS in inverted blue colour scale with $\left(20^{\prime \prime} \times 20^{\prime \prime}\right)$ black contours (eight equally spaced relative contour levels starting from three times $\sigma$, where $\sigma$ denotes the local rms noise). The circles at bottom left corner represents the beam sizes of low-resolution radio maps of LoTSS DR1. The red colour marker "+" indicates the location of the host galaxy. 

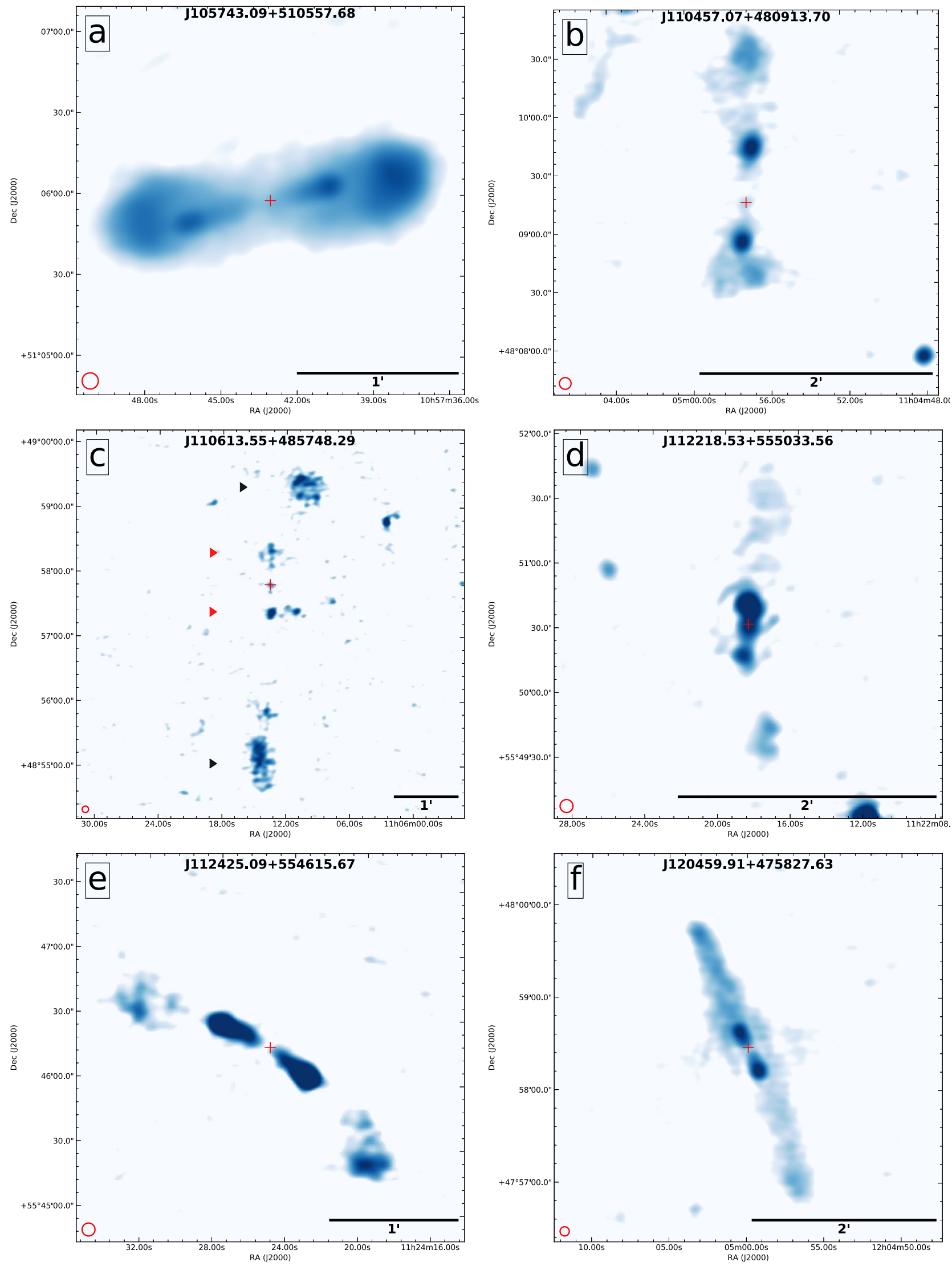

Fig. 11. DDRGs postage-1 (1-6 DDRGs) made using LoTSS DR1 $144 \mathrm{MHz}$ radio image with $6^{\prime \prime} \times 6^{\prime \prime}$ resolution in inverted blue colour scale. Refer to Sect. 4.2.2 for more details. The circle at bottom left corner represents the beam size. The red colour marker "+" indicates the location of the host galaxy. In source J110613.55+485748.29 (sub-image c), the two red markers indicate the inner pair of the DDRG and the black markers indicate the outer pair of the DDRG. 
A\&A 635, A5 (2020)
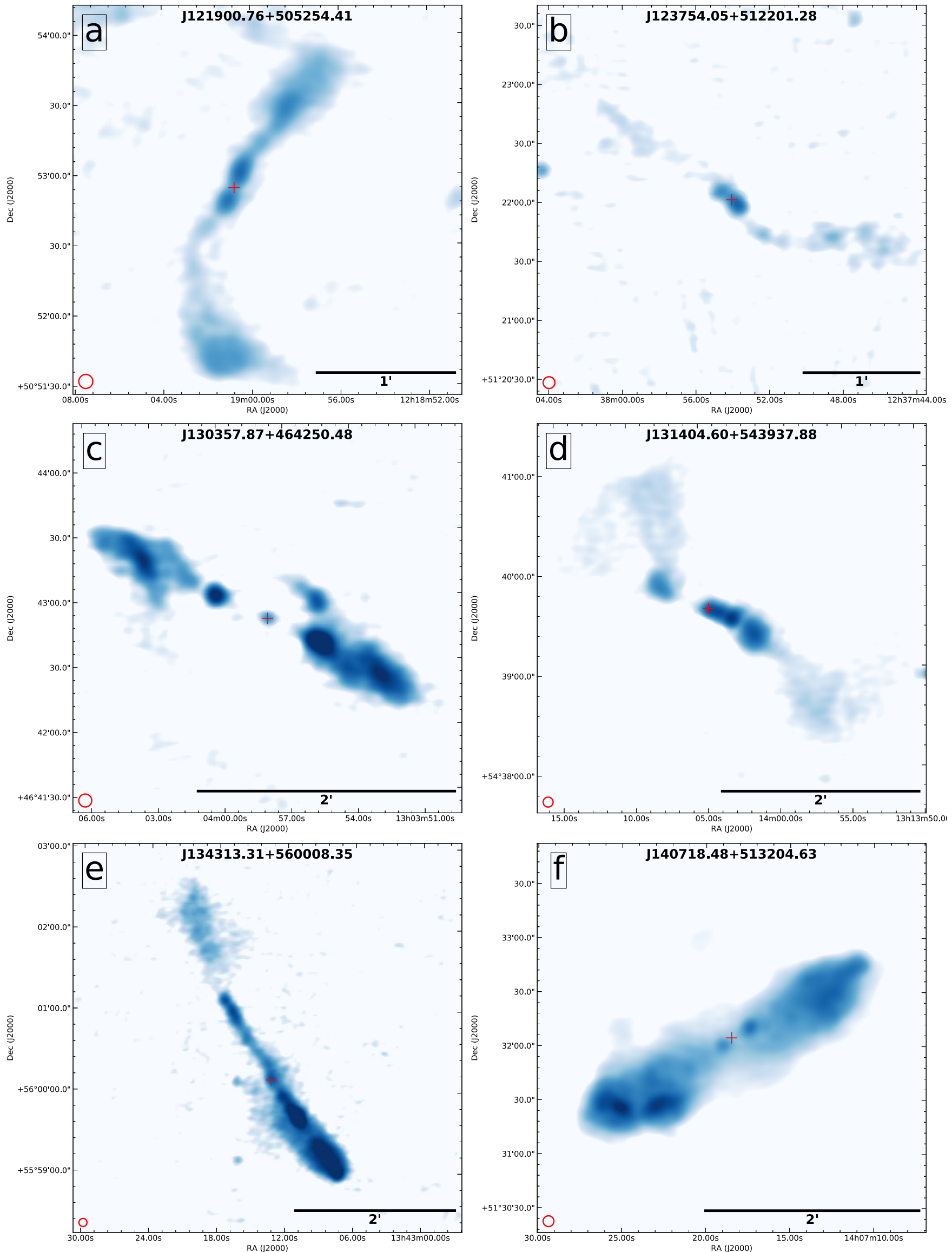

Fig. 12. DDRGs postage-1 (7-12 DDRGs) made using LoTSS DR1 $144 \mathrm{MHz}$ radio image with 6 " $\times 6^{\prime \prime}$ resolution in inverted blue colour scale. Refer to Sect. 4.2.2 for more details. 

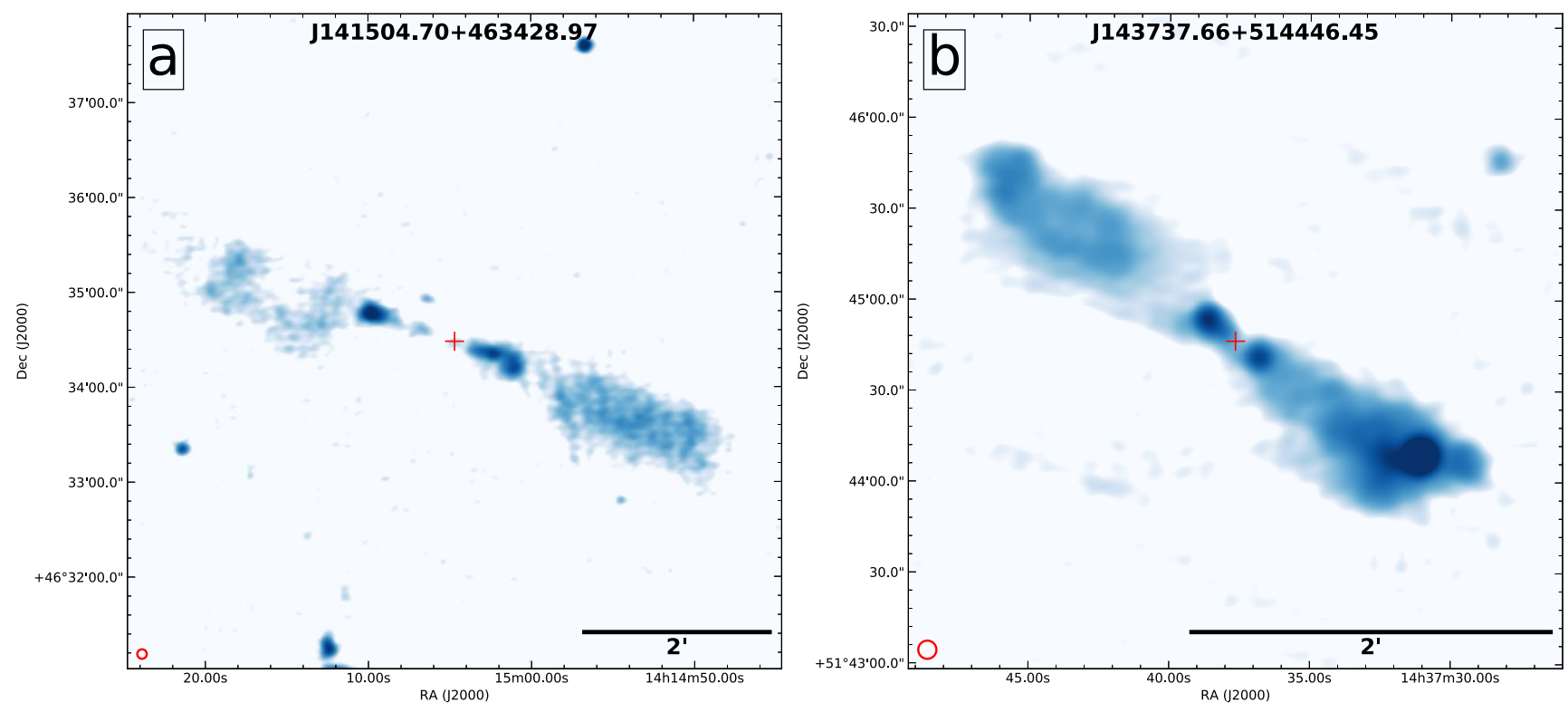

Fig. 13. DDRGs postage-1 (13-14 DDRGs) made using LoTSS DR1 $144 \mathrm{MHz}$ radio image with $6^{\prime \prime} \times 6^{\prime \prime}$ resolution in inverted blue colour scale. Refer to Sect. 4.2.2 for more details.

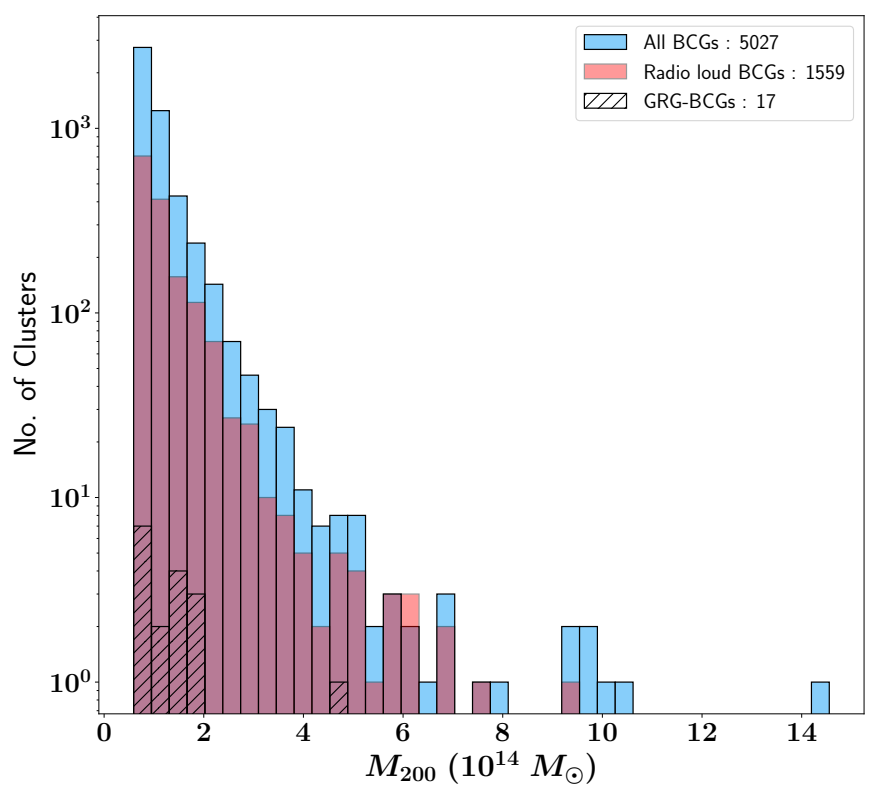

Fig. 14. Distribution of $M_{200}$ (mass of the cluster within $r_{200}$ ) of clusters of galaxies.

Table 1. Short summary of classification of GRGs.

\begin{tabular}{lc}
\hline \hline Classification & No. of objects \\
\hline GRQs & 40 \\
BCGs & 20 \\
FR-II & 215 \\
FR-I & 18 \\
HyMoRS & 6 \\
DDRGs & 14 \\
\hline
\end{tabular}

\section{Summary}

A total of 239 GRGs (Table 1) were found in $\sim 424 \mathrm{deg}^{2}$ area using LoTSS, which is just $\sim 2 \%$ of the area of the total survey that is planned to cover the northern sky. The summary of the paper is as follows.

1. Our sample of 239 GRGs is in the redshift range of $0.1-2.3$, out of which 225 are newly found. This makes it the largest sample discovered to date.

2. About 16\% (40 out of 239) of the sample are hosted by quasars, where three GRQs are above a redshift of 2 .

3. The depth and resolution of LoTSS images has enabled us to find GRGs with low powers of $\sim 10^{24} \mathrm{~W} \mathrm{~Hz}^{-1}$ at $144 \mathrm{MHz}$.

4. We show that the spectral index of GRGs and GRQs is similar to that of their low-sized counterparts (NRGs).

5. The majority $(90 \%)$ of our sample show FR-II-type morphology.

6. We found 14 double-double GRGs, which is $\sim 5 \%$ of our sample. It is currently the largest reporting sample of giant DDRGs.

7. We have found 6 GRGs with HyMoRS morphology, which is very rare.

8. Based on the optical data, we find that none of the GRGs in our sample are hosted by spiral galaxies.

9. At $z<0.55$, at least $\sim 16 \%$ of GRGs lie at the centres (BCGs) of either big galaxy groups or galaxy clusters.

Our sample of 239 GRGs represents a lower number estimate due to the limitation of optical data, which is essential for identifying a host galaxy and its corresponding redshift. Assuming the isotropy and homogeneity of the Universe, if we extrapolate the number of GRGs expected to be found over the final sky coverage of LoTSS ( $\sim 2 \pi$ steradians), then we should be able to find at least $\sim 12000$ GRGs with LoTSS's sensitivity. Upcoming deep optical spectroscopic surveys, such as the WEAVE-LOFAR survey (Smith et al. 2016), will provide crucial data (redshifts) for identifying hundreds or more GRGs.

Acknowledgements. We acknowledge the comments and suggestions of the anonymous referee which has helped us improve the quality of our paper. PD and JB gratefully acknowledge generous support from the Indo-French Center for the Promotion of Advanced Research (Centre Franco-Indien pour la Promotion de la Recherche Avanćee) under programme no. 5204-2 and thank IUCAA 
and IUCAA Radio Physics Lab (RPL) for financial and logistic support. This research has made use of the Dutch national e-infrastructure with support of the SURF Cooperative (e-infra 180169) and the LOFAR e-infra group. The Jülich LOFAR Long Term Archive and the German LOFAR network, are both coordinated and operated by the Jülich Supercomputing Centre (JSC), and computing resources on the Supercomputer JUWELS at JSC were provided by the Gauss Centre for Supercomputing e.V. (www.gauss-centre.eu, grant CHTB00) through the John von Neumann Institute for Computing (NIC). HJR gratefully acknowledge generous support from the European Research Council under the European Unions Seventh Framework Programme (FP/2007-2013)/ERC Advanced Grant NEWCLUSTERS-321271. MJH acknowledges support from the UK Science and Technology Facilities Council [ST/R000905/1]. This research has made use of the University of Hertfordshire high-performance computing facility (https://uhhpc.herts.ac.uk/) and the LOFAR-UK compute facility, located at the University of Hertfordshire and supported by STFC [ST/P000096/1]. HJR and KJD acknowledges support from the ERC Advanced Investigator programme NewClusters 321271. LOFAR, the Low Frequency Array designed and constructed by ASTRON, has facilities in several countries, which are owned by various parties (each with their own funding), and are collectively operated by the International LOFAR Telescope (ILT) foundation under a joint scientific policy. We thank the LOFAR Galaxy Zoo team We gratefully acknowledge the use of Edward (Ned) Wright's online Cosmology Calculator. This research has made use of the NASA Extragalactic Database (NED) which is operated by the Jet Propulsion Laboratory, California Institute of Technology, under contract with the National Aeronautics and Space Administration. This research has also made use of the SIMBAD database, operated at CDS, Strasbourg, France. This publication makes use of data products from the Wide-field Infrared Survey Explorer, which is a joint project of the University of California, Los Angeles, and the Jet Propulsion Laboratory/California Institute of Technology, funded by the National Aeronautics and Space Administration

\section{References}

Amirkhanyan, V. R. 2016, Astrophys. Bull., 71, 384

Amirkhanyan, V. R., Afanasiev, V. L., \& Moiseev, A. V. 2015, Astrophys. Bull., 70, 45

Bagchi, J., Durret, F., Neto, G. B. L., \& Paul, S. 2006, Science, 314, 791

Bagchi, J., Vivek, M., Vikram, V., et al. 2014, ApJ, 788, 174

Becker, R. H., White, R. L., \& Helfand, D. J. 1995, ApJ, 450, 559

Begelman, M. C., Blandford, R. D., \& Rees, M. J. 1984, Rev. Mod. Phys., 56, 255

Bock, D. C.-J., Large, M. I., \& Sadler, E. M. 1999, AJ, 117, 1578

Chambers, K. C., Magnier, E. A., Metcalfe, N., et al. 2016, ArXiv e-prints [arXiv:1612.05560]

Clarke, A. O., Heald, G., Jarrett, T., et al. 2017, A\&A, 601, A25

Colless, M., Dalton, G., Maddox, S., et al. 2001, MNRAS, 328, 1039

Condon, J. J., Cotton, W. D., Greisen, E. W., et al. 1998, AJ, 115, 1693

Cotter, G., Rawlings, S., \& Saunders, R. 1996, MNRAS, 281, 1081

Croston, J. H., Hardcastle, M. J., Mingo, B., et al. 2019, A\&A, 622, A10

Dabhade, P., Gaikwad, M., Bagchi, J., et al. 2017, MNRAS, 469, 2886

Duncan, K. J., Sabater, J., Röttgering, H. J. A., et al. 2019, A\&A, 622, A3

Fanaroff, B. L., \& Riley, J. M. 1974, MNRAS, 167, 31P

Gawroński, M. P., Marecki, A., Kunert-Bajraszewska, M., \& Kus, A. J. 2006, A\&A, 447, 63

Gopal-Krishna, \& Wiita, P. J. 2002, New Astron. Rev., 46, 357

Gruppioni, C., Zamorani, G., de Ruiter, H. R., et al. 1997, MNRAS, 286, 470

Hao, J., McKay, T. A., Koester, B. P., et al. 2010, ApJS, 191, 254

Hardcastle, M. J., Gürkan, G., van Weeren, R. J., et al. 2016, MNRAS, 462 1910

Hart, R. E., Bamford, S. P., Willett, K. W., et al. 2016, MNRAS, 461, 3663

Heald, G. H., Pizzo, R. F., Orrú, E., et al. 2015, A\&A, 582, A123

Hota, A., Sirothia, S. K., Ohyama, Y., et al. 2011, MNRAS, 417, L36

Huchra, J. P., Macri, L. M., Masters, K. L., et al. 2012, ApJS, 199, 26

Hurley-Walker, N., Callingham, J. R., Hancock, P. J., et al. 2017, MNRAS, 464 1146

Intema, H. T., Jagannathan, P., Mooley, K. P., \& Frail, D. A. 2017, A\&A, 598,

Ishwara-Chandra, C. H., \& Saikia, D. J. 1999, MNRAS, 309, 100

Ishwara-Chandra, C. H., Sirothia, S. K., Wadadekar, Y., et al. 2010, MNRAS 405,436

Isobe, N., \& Koyama, S. 2015, PASJ, 67, 77

Jennison, R. C., \& Das Gupta, M. K. 1953, Nature, 172, 996

Jones, D. H., Read, M. A., Saunders, W., et al. 2009, MNRAS, 399, 683

Kaiser, N., Aussel, H., Burke, B. E., et al. 2002, Proc. SPIE, 4836, 154

Kaiser, N., Burgett, W., Chambers, K., et al. 2010, Proc. SPIE, 7733, 77330E
Kapahi, V. K., Athreya, R. M., van Breugel, W., et al. 1998, ApJS, 118, 275 Kapińska, A. D., Terentev, I., Wong, O. I., et al. 2017, AJ, 154, 253 Komberg, B. V., \& Pashchenko, I. N. 2009, Astron. Rep., 53, 1086 Kozieł-Wierzbowska, D., \& Stasińska, G. 2011, MNRAS, 415, 1013 Kronberg, P. P., Colgate, S. A., Li, H., \& Dufton, Q. W. 2004, ApJ, 604, L77

Kuligowska, E., \& Kuźmicz, A. 2018, XXXVIII Polish Astronomical Society Meeting, 82

Kuźmicz, A., Jamrozy, M., Kozieł-Wierzbowska, D., et al. 2017, MNRAS, 471, 3806

Kuźmicz, A., Jamrozy, M., Bronarska, K., et al. 2018, ApJS, 238, 9

Lacy, M., Rawlings, S., Saunders, R., et al. 1993, MNRAS, 264, 721

Laing, R. A., Riley, J. M., \& Longair, M. S. 1983, MNRAS, 204, 151

Lara, L., Cotton, W. D., Feretti, L., et al. 2001, A\&A, 370, 409

Lara, L., Giovannini, G., Cotton, W. D., et al. 2004, A\&A, 421, 899

Ledlow, M. J., \& Owen, F. N. 1996, AJ, 112, 9

Lopes, P. A. A. 2007, MNRAS, 380, 1608

Lynden-Bell, D. 1969, Nature, 223, 690

Machalski, J. 1998, A\&AS, 128, 153

Machalski, J., Jamrozy, M., \& Zola, S. 2001, A\&A, 371, 445

Machalski, J., Koziel-Wierzbowska, D., \& Jamrozy, M. 2007, Acta Astron., 57, 227

Mack, K.-H., Klein, U., O’Dea, C. P., Willis, A. G., \& Saripalli, L. 1998, A\&A, 329,431

Mahatma, V. H., Hardcastle, M. J., Williams, W. L., et al. 2019, A\&A, 622 , A13

Malarecki, J. M., Jones, D. H., Saripalli, L., Staveley-Smith, L., \& Subrahmanyan, R. 2015, MNRAS, 449, 955

McGilchrist, M. M., \& Riley, J. M. 1990, MNRAS, 246, 123

Mohan, N., \& Rafferty, D. 2015, Astrophysics Source Code Library [record ascl:1502.007]

Mahony, E. K., Morganti, R., Prandoni, I., et al. 2016, MNRAS, 463, 2997

Nandi, S., \& Saikia, D. J. 2012, Bull. Astron. Soc. India, 40, 12

Nandi, S., Saikia, D. J., Roy, R., et al. 2019, MNRAS, 486, 5158

Nilsson, K. 1998, A\&AS, 132, 31

Oort, M. J. A., Steemers, W. J. G., \& Windhorst, R. A. 1988, A\&AS, 73, 103

O'Sullivan, S. P., Machalski, J., Van Eck, C. L., et al. 2019, A\&A, 622, A16

Pâris, I., Petitjean, P., Aubourg, É., et al. 2018, A\&A, 613, A51

Paul, S., John, R. S., Gupta, P., \& Kumar, H. 2017, MNRAS, 471, 2

Planck Collaboration XIII. 2016, A\&A, 594, A13

Prescott, M., Whittam, I. H., Jarvis, M. J., et al. 2018, MNRAS, 480, 707

Richards, G. T., Myers, A. D., Gray, A. G., et al. 2009, ApJS, 180, 67

Richards, G. T., Myers, A. D., Peters, C. M., et al. 2015, ApJS, 219, 39

Rengelink, R. B., Tang, Y., de Bruyn, A. G., et al. 1997, A\&AS, 124, 259

Rentería Macario, J., \& Andernach, H. 2017, ArXiv e-prints [arXiv:1710.10731]

Rottgering, H. J. A., Wieringa, M. H., Hunstead, R. W., \& Ekers, R. D. 1997, MNRAS, 290, 577

Saikia, D. J., \& Jamrozy, M. 2009, Bull. Astron. Soc. India, 37, 63

Saikia, D. J., Thomasson, P., Jackson, N., Salter, C. J., \& Junor, W. 1996, MNRAS, 282, 837

Safouris, V., Subrahmanyan, R., Bicknell, G. V., \& Saripalli, L. 2009, MNRAS, 393, 2

Saripalli, L., \& Malarecki, J. M. 2015, The Many Facets of Extragalactic Radio Surveys: Towards New Scientific Challenges, 44

Saripalli, L., Hunstead, R. W., Subrahmanyan, R., \& Boyce, E. 2005, AJ, 130, 896

Schoenmakers, A. P., de Bruyn, A. G., Röttgering, H. J. A., van der Laan, H., \& Kaiser, C. R. 2000, MNRAS, 315, 371

Schoenmakers, A. P., de Bruyn, A. G., Röttgering, H. J. A., \& van der Laan, H. 2001, A\&A, 374, 861

Sebastian, B., Ishwara-Chandra, C. H., Joshi, R., et al. 2018, MNRAS, 473, 4926 Shimwell, T. W., Röttgering, H. J. A., Best, P. N., et al. 2017, A\&A, 598, A104

Shimwell, T. W., Tasse, C., Hardcastle, M. J., et al. 2019, A\&A, 622, A1

Smith, D. J. B., Best, P. N., Duncan, K. J., et al. 2016, SF2A-2016: Proceedings of the Annual Meeting of the French Society of Astronomy and Astrophysics, 271

Solovyov, D. I., \& Verkhodanov, O. V. 2014, Astrophys. Bull., 69, 141 Subrahmanyan, R., Saripalli, L., \& Hunstead, R. W. 1996, MNRAS, 279, 257

Thwala, S. A., Shafi, N., Colafrancesco, S., et al. 2019, MNRAS, 485, 1938 van Weeren, R. J., Brüggen, M., Röttgering, H. J. A., et al. 2011, A\&A, 533, A35

Wen, Z. L., Han, J. L., \& Liu, F. S. 2012, ApJS, 199, 34

Wiita, P. J., Rosen, A., Gopal-Krishna, \& Saripalli, L. 1989, Hot Spots in Extragalactic Radio Sources, 327, 173

Williams, W. L., Hardcastle, M. J., Best, P. N., et al. 2019, A\&A, 622, A2

Willis, A. G., Strom, R. G., \& Wilson, A. S. 1974, Nature, 250, 625

Wright, E. L., Eisenhardt, P. R. M., Mainzer, A. K., et al. 2010, AJ, 140 , 1868

York, D. G., Adelman, J., Anderson, Jr., J. E., et al. 2000, AJ, 120, 1579 


\section{Appendix A: Additional tables and figures}

Table A.1. 239 GRGs found from the LoTSS.

\begin{tabular}{|c|c|c|c|c|c|c|c|c|c|c|c|c|}
\hline Sr.No & $\begin{array}{c}\text { RA } \\
(\mathrm{HMS}) \\
(2)\end{array}$ & $\begin{array}{c}\text { Dec } \\
(\mathrm{DMS}) \\
(3)\end{array}$ & Class & (5) & $\begin{array}{l}\text { Size } \\
\left({ }^{\prime \prime}\right) \\
(6)\end{array}$ & $\begin{array}{l}\text { Size } \\
(\mathrm{Mpc}) \\
(7)\end{array}$ & $\begin{array}{c}S_{144 \mathrm{MHz}} \\
(\mathrm{mJy}) \\
(8)\end{array}$ & $\begin{array}{c}P_{144 \mathrm{MHz}} \\
\left(10^{26} \mathrm{~W} \mathrm{~Hz}^{-1}\right) \\
(9)\end{array}$ & $\begin{array}{l}\alpha_{144}^{1400} \\
(10)\end{array}$ & $\begin{array}{l}\text { FR } \\
\text { Type } \\
(11)\end{array}$ & $\begin{array}{c}r \\
\mathrm{Mag} \\
(12)\end{array}$ & Ref. \\
\hline 1 & 5705.43 & 32624.79 & Q & $2 \pm 0.000$ & 153 & 0.92 & $1485 \pm 297$ & $11.00 \pm 2.20$ & $0.69 \pm 0.09$ & II & $19.48 \pm 0.03$ & - \\
\hline 2 & 5709.25 & $10-104$ & $\mathrm{G}$ & 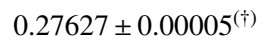 & 439 & & $1062 \pm 212$ & $2.55 \pm$ & $0.79 \pm 0.09$ & II & $18.01 \pm 0.01$ & - \\
\hline 3 & 571 , & 2 & Q & 1 & 91 & & & $1.84 \pm$ & $0.64 \pm 0.11$ & II & $8.86 \pm 0.01$ & - \\
\hline 4 & 105721.22 & +531422.42 & G & $0.33645 \pm 0.00011^{(\dagger)}$ & 145 & 0.72 & & $0.04 \pm 0.02$ & $<0.63$ & II & $18.36 \pm 0.02$ & - \\
\hline 5 & 105725.96 & +492900.31 & G & $0.47614 \pm 0.00022^{(\dagger)}$ & 157 & 0.96 & $94 \pm 19$ & $0.81 \pm 0.16$ & $0.86 \pm 0.10$ & II & $19.76 \pm 0.04$ & - \\
\hline 6 & 105743.09 & +510557.68 & G & $0.46269 \pm 0.00004^{(\dagger)}$ & 126 & 0.76 & $657 \pm 131$ & $5.22 \pm 1.05$ & $0.85 \pm 0.09$ & II & $20.59 \pm 0.05$ & - \\
\hline 7 & 105817.90 & +514017.70 & G & $0.41497 \pm 0.00003^{(\dagger)}$ & 330 & 1.86 & $298 \pm 88$ & $1.77 \pm 0.53$ & $0.74 \pm 0.22$ & II & $19.62 \pm 0.03$ & (8) \\
\hline 8 & 110147.58 & +464911.20 & G & $0.68096 \pm 0.00017^{(\dagger)}$ & 150 & 1.09 & $532 \pm 106$ & $10.70 \pm 2.15$ & $0.88 \pm 0.09$ & II & & (3) \\
\hline 9 & 110159.17 & +464534.19 & G & & 127 & & & & & $\mathrm{H}$ & & - \\
\hline 10 & 110433.11 & +46422 & G & & 305 & & & & & I & & (9) \\
\hline 11 & 11045 & +48091 & 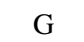 & $1 \pm 0.00$ & 137 & & & & 0.75 & II & & - \\
\hline 12 & 11051 & +544 & $\mathrm{C}$ & 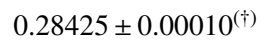 & 169 & & & & 10 & & & - \\
\hline 13 & 11061 & +485 & -3 & 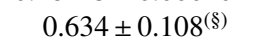 & 292 & & & & & 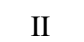 & & - \\
\hline 14 & 110 & & 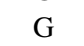 & & 157 & & & & & II & & - \\
\hline 15 & 1108 & & 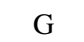 & $c^{2}$ & 103 & & & & & II & & - \\
\hline 16 & & & 0 & & 85 & & & & & II & & - \\
\hline 17 & 21 & 1 & G & & 183 & & & & 12 & II & & - \\
\hline 18 & 11093 & +510 & 0 & & 106 & & & & - & II & & (2) \\
\hline 19 & 11093 & +531 & G & & 230 & & & & - & II & & - \\
\hline 20 & 111011.02 & +533058.74 & 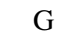 & $0.58483 \pm 0.00018^{(\dagger)}$ & 142 & 0.9 & 56 & & - & II & & - \\
\hline 21 & 111259.52 & +494227.11 & G & $0502 \quad 0122(8)$ & 244 & 1.5 & 22 & 26 & 0 & II & & - \\
\hline 22 & 111331.45 & +462216.04 & G & $0.589 \pm 0.14$ & 112 & 0.7 & 34 & 2 & $0.80 \pm$ & II & 10 & - \\
\hline 23 & 111400.10 & +532216.57 & G & $0.727 \pm 0.116^{(\S)}$ & & & & & & II & & - \\
\hline 24 & 111529.60 & & 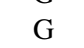 & & 10 & & & & $77 \pm 0$ & II & & - \\
\hline 25 & 111 & & $\mathrm{C}$ & & 34 & & & & - & III & & - \\
\hline 26 & 112 & & & & 10 & & & & 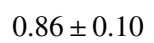 & $-\pi$ & & - \\
\hline 27 & 1 & & $\mathrm{C}$ & & 45 & & & & & 2 & & - \\
\hline 28 & 35 & 4 & 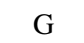 & () & 132 & 0 . & & & $0.61 \pm 0$ & II & .04 & - \\
\hline 29 & 11 & $5-6$ & $C^{2}+2$ & $7^{(\dagger)}$ & 126 & 1. & 5 & & - & II & 18 & - \\
\hline 30 & 11242 & +55 & G & $0.80 \mathrm{~s}$ & 146 & 1. & & & $7 \pm 0$ & II & .22 & - \\
\hline 31 & 112429.98 & +463523.68 & Q & $0.91500 \pm 0.00013^{(\dagger)}$ & 88 & 0.7 & 22,7 & $1 \pm 0.23$ & $0.58 \pm 0.10$ & II & $20.12 \pm 0.03$ & - \\
\hline 32 & 112435.86 & +490325.92 & G & $0.47934 \pm 0.00010^{(\dagger)}$ & 121 & & $120 \pm 24$ & & - & II & $20.10 \pm 0.04$ & - \\
\hline 33 & 112629.95 & +490137.16 & G & $0.660 \pm 0.139^{(\S)}$ & 111 & 0.8 & 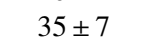 & $0.62 \pm$ & $0.76 \pm 0.11$ & II & $21.67 \pm 0.12$ & - \\
\hline 34 & 112639.89 & +533427.20 & G & $0.64523 \pm 0.00024^{(\dagger)}$ & 125 & 0.8 & $79 \pm 16$ & $1.30 \pm 0.26$ & - & II & $20.49 \pm 0.08$ & - \\
\hline 35 & 112713.18 & +511326.35 & G & & 202 & 1. & & & $0.60 \pm 0$ & I & & - \\
\hline 36 & 112742.06 & +521944.68 & $C_{0}$ & & 11 & & & & & II & & - \\
\hline 37 & & & 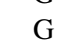 & & 18 & & & & - & II & & - \\
\hline 38 & & & 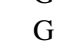 & & & & & & & 1 & & - \\
\hline 39 & & & G & 0.2 & 20 & & & & & 11 & & - \\
\hline 40 & 113250.67 & +505704.68 & G & $0.35857 \pm 0.00010^{(\dagger)}$ & 138 & 0.71 & $63 \pm 13$ & $0.26 \pm 0.05$ & $0.65 \pm 0.10$ & II & $18.53 \pm 0.01$ & - \\
\hline 41 & 113421.89 & +563413.28 & G & $0.626 \pm 0.092^{(\S)}$ & 227 & 1.59 & $21 \pm 4$ & $0.35 \pm 0.14$ & $0.92 \pm 0.16$ & II & $20.98 \pm 0.08$ & - \\
\hline 42 & 113435.48 & +460800.45 & G & $0.704 \pm 0.163^{(\S)}$ & 300 & 2.21 & $41 \pm 8$ & $0.90 \pm 0.54$ & $0.87 \pm 0.16$ & II & $21.39 \pm 0.08$ & - \\
\hline 43 & 113503.20 & +482612.12 & G & $22597 \pm 0.00006^{(\dagger)}$ & 202 & 0.76 & $31 \pm 26$ & $0.20 \pm 0.04$ & $0.78 \pm 0.09$ & II & $17.59 \pm 0.01$ & - \\
\hline 44 & 113931.77 & +472124.3 & G & $0.51791 \pm 0.00012^{(\dagger)}$ & 312 & 2.00 & $126 \pm 25$ & $1.23 \pm 0.25$ & $0.70 \pm 0.09$ & II & $20.32 \pm 0.05$ & - \\
\hline
\end{tabular}

Notes. Columns (2) RA and (3) Dec are the right ascension and declination (J2000.0 epoch), which indicate the centre of the host galaxies of the GRG and GRQs from either SDSS or Pan-STARRS. Column (4) "Class" represents the type of host of the GRG - G: galaxy, Q: quasar; and $Q_{\mathrm{c}}$ : quasar candidate. In Col. (5), $z$ (redshift) marked with ${ }^{\left({ }^{\star}\right)}$ represents spectroscopic redshift from SDSS, ${ }^{(\star)}$ represents photometric redshift from SDSS, and $\left.{ }^{(}\right)$indicates redshifts from VAC. The one marked with superscript "a”" refers to redshift obtained from O'Sullivan et al. (2019) (spectroscopic) and "b" from Lopes (2007) (photometric), owing to higher accuracy when compared to SDSS or VAC. Columns (6) and (7) are angular size and projected linear size of the source. Columns (8) and (9) are the integrated flux of sources at $144 \mathrm{MHz}$ and its corresponding radio power. Column (10) $\alpha_{144}^{1400}$ is the integrated spectral index computed between $1400 \mathrm{MHz}$ (NVSS) flux and $144 \mathrm{MHz}$ (LoTSS) flux. Rows marked with "-" in Col. (10) are blended sources for which we do not present a spectral index. Column (11) indicates the morphological type of the GRG: I represents FR-I type, II represents FR-II type and III represents HyMoRS. Column (12) represents the $r$ band magnitude of the host galaxies of the GRGs obtained from SDSS. For sources 162 and 233, in the absence of reliable magnitudes from SDSS, we have given $r$ band magnitudes from PAN-STARRS. The last column (Ref.) shows references (see end of the table) for the GRGs already known in literature.

References. (1) Amirkhanyan et al. (2015); (2) Amirkhanyan (2016); (3) Cotter et al. (1996); (4) Kozieł-Wierzbowska \& Stasińska (2011); (5) Machalski (1998); (6) Machalski et al. (2001); (7) Nilsson (1998); (8) Rentería Macario \& Andernach (2017); (9) Thwala et al. (2019); (10) Kuźmicz et al. (2018). 
Table A.1. continued.

\begin{tabular}{|c|c|c|c|c|c|c|c|c|c|c|c|c|}
\hline Sr.No & $\begin{array}{c}\text { RA } \\
(\mathrm{HMS}) \\
(2) \\
\end{array}$ & $\begin{array}{c}\text { Dec } \\
(\mathrm{DMS}) \\
(3)\end{array}$ & Class & (5) & $\begin{array}{c}\text { Size } \\
\left({ }^{\prime \prime}\right) \\
(6) \\
\end{array}$ & $\begin{array}{l}\text { Size } \\
(\mathrm{Mpc}) \\
(7)\end{array}$ & $\begin{array}{c}S_{144 \mathrm{MHz}} \\
(\mathrm{mJy}) \\
(8)\end{array}$ & $\begin{array}{c}P_{144 \mathrm{MHz}} \\
\left(10^{26} \mathrm{~W} \mathrm{~Hz}^{-1}\right) \\
(9)\end{array}$ & $\begin{array}{l}\alpha_{144}^{1400} \\
(10) \\
\end{array}$ & $\begin{array}{l}\text { FR } \\
\text { Type } \\
(11) \\
\end{array}$ & $\begin{array}{c}r \\
\text { Mag } \\
(12) \\
\end{array}$ & (13) \\
\hline 45 & 4305.55 & 2726.89 & $\mathrm{Q}$ & \pm 0 & 86 & 0.75 & 0 & 4 & 0 & II & 15 & - \\
\hline 46 & 114814.98 & +5716.49 & G & $22679 \pm 0.0000$ & 202 & 0.76 & & $0.03=$ & $51 \pm 0.11$ & I & .01 & - \\
\hline 47 & 115159.9 & +495056.11 & Q & $39148 \pm 0.00017^{(\dagger)}$ & 96 & 0.77 & $1015+203$ & $3660-$ & $0.78 \pm 0.09$ & II & $8.38 \pm 0.01$ & 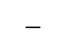 \\
\hline 48 & 115216.89 & +462451.37 & G & $0.44454 \pm 0.02004^{(\star)}$ & 148 & 0.87 & & & $0.56 \pm 0.10$ & II & $19.68 \pm 0.03$ & 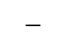 \\
\hline 49 & 115839.12 & +465545.35 & G & $0.65974 \pm 0.00023^{(\dagger)}$ & 128 & 0.92 & $18 \pm 4$ & $0.36 \pm 0.07$ & $<1.08$ & II & $21.61 \pm 0.13$ & - \\
\hline 50 & 120118.14 & +463241.98 & G & $0.638 \pm 0.088^{(\S)}$ & 129 & 0.91 & $111 \pm 22$ & $1.87 \pm 0.73$ & $0.83 \pm 0.10$ & II & $21.82 \pm 0.15$ & - \\
\hline 51 & 120122.84 & +492537.14 & G & $0.205 \pm 0.045^{(\S)}$ & 345 & 1.20 & $1946 \pm 389$ & $2.56 \pm 1.35$ & $1.09 \pm 0.09$ & I & $18.14 \pm 0.01$ & - \\
\hline 52 & 120206.86 & +514134.63 & $\mathrm{G}$ & $0.61278 \pm 0.00028^{(\dagger)}$ & 141 & 0.98 & $87 \pm 17$ & $1.27 \pm 0.26$ & - & II & $21.11 \pm 0.10$ & - \\
\hline 53 & 120459.91 & +475827.63 & $\mathrm{G}$ & $0.52963 \pm 0.08487^{(\star)}$ & 192 & 1.24 & & & $0.89 \pm 0.09$ & II & & - \\
\hline 54 & 120528.50 & +561342.32 & $\mathrm{G}$ & & 118 & 0.86 & & & & 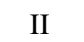 & & - \\
\hline 55 & 120546.98 & +50485 & $\mathrm{G}$ & & 160 & 1.1 & & & & H & & - \\
\hline 56 & 120826.14 & 17152 & $\mathrm{G}$ & $0.56407 \pm 0.00$ & 113 & 0 . & & & - & II & & - \\
\hline 57 & 12104 & 90 & $\mathrm{G}$ & $(\dagger)$ & 119 & 0 . & & & 9 & & & 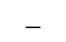 \\
\hline 58 & 12105 & 77 & G & $\star$ ^) & 110 & 0 . & & & & 1 & & - \\
\hline 59 & 12114 & 11 & $\mathrm{G}$ & & 143 & & & & & & & - \\
\hline 60 & 12130 & 38 & Q & & 118 & 0.8 & & & & II & & - \\
\hline 61 & & 1 & $\mathrm{G}$ & †) & 277 & & & & - & I & & - \\
\hline 62 & 12170 & 33 & $\mathrm{G}$ & & 183 & & & & $9 \pm 0$ & I & & - \\
\hline 63 & 121707.40 & +485822.48 & $\mathrm{G}$ & & 136 & 084 & & & - & I & & - \\
\hline 64 & 121818.15 & +532721.33 & G & $056754+0$ & 183 & 1.23 & $1270=$ & $16.00 \pm$ & $0.81 \pm 0.09$ & $\mathrm{I}$ & & - \\
\hline 65 & 121849.88 & +502617.59 & $\mathrm{G}$ & $2^{(\dagger)}$ & 210 & 0.7 & & & $0.67 \pm$ & -1 & & (6) \\
\hline 66 & 121900.76 & +505254.41 & $\mathrm{G}$ & $0.38509 \pm 0.00008^{(\dagger)}$ & 142 & 0.7 & & & 10 & I & & - \\
\hline 67 & 121935.92 & 16502 & $\mathrm{G}$ & 0. & 114 & 0 . & & & - & II & & - \\
\hline 68 & 121952.32 & 472058.49 & Q & & 241 & & & & & & & - \\
\hline 69 & 121952.55 & 465425.74 & $\mathrm{Q}$ & 1.85 & - & & & & & & & - \\
\hline 70 & 07.85 & 55 & $\mathrm{G}$ & & & & & & & & & - \\
\hline 71 & 13 & 89 & $\mathrm{G}$ & & 255 & & & & & & & - \\
\hline 72 & & 32 & G & & 354 & & & & & & & - \\
\hline 73 & & & $\mathrm{G}$ & & 111 & & & & & 1 & & - \\
\hline 74 & & 40 & $\mathrm{G}$ & \$) & 95 & $07-2-3-2-1$ & & & & II & & - \\
\hline 75 & & 95 & G & $99^{(\dagger)}$ & 206 & 1 & & & & I & & - \\
\hline 76 & 1225 & 93 & $\mathrm{G}$ & & 164 & 116 & & & & & & - \\
\hline 77 & 2558.20 & 30938.38 & $\mathrm{G}$ & & 147 & 1. & & & 09 & II & 15 & - \\
\hline 78 & 122826.35 & +523101.10 & $\mathrm{G}$ & (\$) & 157 & 0.9 & & & 000 & 11 & & - \\
\hline 79 & 122936.25 & +501304.65 & $\mathrm{G}$ & $.38357 \pm 0.00007^{(\dagger)}$ & 198 & 1.0 & & 5 & & I & .02 & - \\
\hline 80 & 122959.59 & +533247.04 & $\mathrm{G}$ & $0.52300 \pm 0.05280^{(\star)}$ & 137 & 0.8 & 9 & 1 & $0.90 \pm 0.09$ & 11 & & - \\
\hline 81 & 123014.05 & & $\mathrm{G}$ & & 20 & & & & & II & & - \\
\hline 82 & & & $\mathrm{G}$ & $4 \pm 0.00006^{(\dagger)}$ & & & & & & & & - \\
\hline 83 & & & $\mathrm{G}$ & & & & & & 9 & & & - \\
\hline 84 & & & 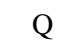 & & 10 & & & & - & & & 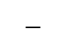 \\
\hline 85 & & & G & & 683 & 3 & & & $5 \pm 0$ & & & - \\
\hline 86 & .77 & 30 & G & ) & 105 & 0.7 & \pm 17 & & - & II & 10 & - \\
\hline 87 & 123648.37 & 460405.91 & G & (\$) & 125 & 0.8 & $268 \pm 54$ & & $0.75 \pm 0$ & 11 & 10 & - \\
\hline 88 & 123754.05 & 512201.28 & $\mathrm{G}$ & $18^{(\dagger)}$ & 164 & 0.89 & 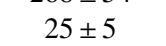 & & - & II & .03 & 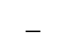 \\
\hline 89 & 123807.78 & +532555.91 & $\mathrm{Q}$ & $0.34680 \pm 0.00004^{(\dagger)}$ & 157 & 0.80 & $455 \pm 91$ & $1.73 \pm 0.35$ & $0.61 \pm 0.09$ & II & $17.29 \pm 0.01$ & - \\
\hline 90 & 123933.21 & 500708.01 & $\mathrm{Q}$ & $1500 \pm 0.00024^{(\dagger)}$ & 89 & 0.75 & $3 \pm 1$ & $2.32 \pm 0.52$ & $<1.39$ & II & $18.49 \pm 0.01$ & - \\
\hline 91 & 124012.46 & +533437.25 & $\mathrm{Q}$ & $0.29300 \pm 0.00003^{(\dagger)}$ & 164 & 0.7 & $27 \pm 30$ & $4.15 \pm 0.83$ & $0.76 \pm 0.09$ & II & $18.52 \pm 0.01$ & (1) \\
\hline 92 & 124031.72 & +485936.89 & $\mathrm{G}$ & $53607 \pm 0.04030^{(\star)}$ & 136 & 0.8 & $605 \pm 121$ & $6.35 \pm 1$ & $0.70 \pm 0.09$ & II & $21.17 \pm 0.08$ & - \\
\hline 93 & 124142.34 & 513514.32 & $\mathrm{G}$ & $0.52951 \pm 0.00011^{(\dagger)}$ & 161 & 1. & & & & II & & - \\
\hline 94 & & & $\mathrm{G}$ & & 11 & & & & & II & & - \\
\hline 95 & & & $\mathrm{G}$ & & & & & & & I & & - \\
\hline 96 & & & $\mathrm{G}$ & & & & & & & & & - \\
\hline 97 & 124913.20 & 68 & G & 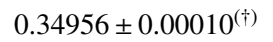 & & 0.7 & & & 9 & I & & \\
\hline 98 & 125142.04 & 03424.65 & G & $(\dagger)$ & 15 & 0.99 & $\tilde{\sigma}$ & & $0 \circ \sigma_{-}$ & & .03 & (5) \\
\hline 99 & 125624.74 & +552825.99 & G & $0.808 \pm 0.179^{(\S)}$ & 113 & 0.87 & $1765 \pm 353$ & $55.50 \pm 32.10$ & $0.94 \pm 0.09$ & II & $21.01 \pm 0.12$ & - \\
\hline 100 & 125716.35 & 511758.11 & Q & $.52582 \pm 0.00004^{(\dagger)}$ & 170 & 1.09 & $96 \pm 19$ & & $0.74 \pm 0.10$ & 11 & $17.69 \pm$ & - \\
\hline 101 & 125821.37 & 42029.35 & $\mathrm{G}$ & $0.612 \pm 0.099^{(\S)}$ & 143 & 0.99 & & $7.64 \pm$ & - & II & $21.11 \pm 0.09$ & - \\
\hline 102 & 125950.83 & +530709.03 & U & $60663 \pm 0.04364^{(\star)}$ & 105 & 0.73 & s & $1.06 \pm$ & $0.94 \pm 0$ & II & $20.93 \pm 0.09$ & - \\
\hline
\end{tabular}


Table A.1. continued.

\begin{tabular}{|c|c|c|c|c|c|c|c|c|c|c|c|c|}
\hline Sr.No & $\begin{array}{c}\text { RA } \\
(\mathrm{HMS}) \\
(2) \\
\end{array}$ & $\begin{array}{c}\text { Dec } \\
(\mathrm{DMS}) \\
(3)\end{array}$ & Class & (5) & $\begin{array}{l}\text { Size } \\
\left({ }^{\prime \prime}\right) \\
(6) \\
\end{array}$ & $\begin{array}{l}\text { Size } \\
(\mathrm{Mpc}) \\
(7)\end{array}$ & $\begin{array}{c}S_{144 \mathrm{MHz}} \\
(\mathrm{mJy}) \\
(8) \\
\end{array}$ & $\begin{array}{c}P_{144 \mathrm{MHz}} \\
\left(10^{26} \mathrm{~W} \mathrm{~Hz}^{-1}\right) \\
(9)\end{array}$ & $\begin{array}{r}\alpha_{144}^{1400} \\
(10) \\
\end{array}$ & $\begin{array}{l}\text { FR } \\
\text { Type } \\
(11) \\
\end{array}$ & $\begin{array}{c}r \\
\text { Mag } \\
(12) \\
\end{array}$ & (13) \\
\hline 103 & 0134.99 & 40809.21 & $\vec{U}$ & & & & & & & 11 & & (1) \\
\hline 104 & 0331.08 & 35948.65 & G & & 248 & 007 & & & & & & - \\
\hline 105 & 0332.19 & 22001.98 & G & 7000 & 165 & 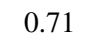 & 7 & $.10=-2 y$ & 99 & $\mathrm{I}$ & 01 & 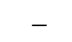 \\
\hline 106 & 37 & 18 & G & ( & 159 & & & & & II & & 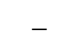 \\
\hline 107 & 30 & 0410 & Q & & 122 & 0.98 & & & & II & & - \\
\hline 108 & 130521.32 & 95142.36 & Q & & 88 & 0.75 & & $44.80 \pm 9$ & & II & & 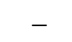 \\
\hline 109 & 130635.08 & 553644.43 & G & & 136 & 09 & & & $74 \pm$ & II & & 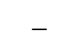 \\
\hline 110 & 130748.36 & 61146.96 & G & & 120 & & & & - & II & & - \\
\hline 111 & 130925.74 & $5348 \quad 17.26$ & G & & 118 & 007 & & & & II & & - \\
\hline 112 & 131028.87 & 21340.43 & G & & 197 & & & & & II & & 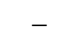 \\
\hline 113 & 1 & & Q & & & & & & & II & & 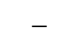 \\
\hline 114 & 1 & & G & & & & & & & & & \\
\hline 115 & & & a $>$ & & & & & & & & & 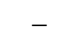 \\
\hline 116 & & & G & & & & & & 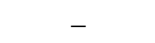 & & & - \\
\hline 117 & & & G & & & & & & 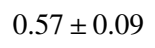 & & & - \\
\hline 118 & & & $\mathrm{C}$ & & & & & & & & & - \\
\hline 119 & & & G & & 262 & & & & & II & & - \\
\hline 120 & & & Q & & 113 & & & & - & II & & 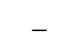 \\
\hline 121 & 07 & & G & & 326 & & & & - & II & & - \\
\hline 122 & 36 & 88 & G & & 180 & & & & - & II & & - \\
\hline 123 & 1323 & 39 & $\mathrm{G}$ & & 136 & & & & & II & & - \\
\hline 124 & 19 & 31 & G & & 162 & 0 & & & & II & & - \\
\hline 125 & 132 & 3 & G & & 07 & & & & & II & & - \\
\hline 126 & 132 & & $G$ & & & & & & & II & & (7) \\
\hline 127 & & & Q & & & & & & & - & & 政 \\
\hline 128 & 1326 & & Q & & & & & & & & & - \\
\hline 12 & & & & & & & & & & & & 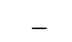 \\
\hline 13 & & & & & & & & & & & & - \\
\hline 13 & & & & & & & & & & & & - \\
\hline 132 & & & G & & 15 & & & & & II & & - \\
\hline 133 & & & $G$ & & & & & & & II & & - \\
\hline 134 & & & $G$ & & 122 & & & & & II & & - \\
\hline 135 & & & $G$ & & 174 & & & & & II & & - \\
\hline 136 & & & $C_{1}>$ & & 173 & & & & - & & & - \\
\hline 137 & & & O & & 01 & & & 0 & 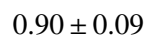 & II & & (4) \\
\hline 138 & 3418.63 & & Q & & & & & 90 & - & II & & - \\
\hline 139 & & & G & & & & & & & II & & - \\
\hline 140 & & & Q & & & & & & - & II & & - \\
\hline 141 & & & G & & & & & & & II & & - \\
\hline 142 & & & 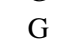 & & & & & & & & & - \\
\hline 14 & & & & & & & & & - & & & - \\
\hline 144 & & & & & & & & & 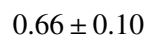 & & & - \\
\hline 145 & & & 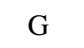 & & & & & & & 11 & & - \\
\hline 146 & & & ( & & 36 & & & & & II & & - \\
\hline 147 & & & 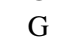 & & 245 & & & & & II & & - \\
\hline 148 & & & G & & 202 & & & & & II & & - \\
\hline 149 & 1.82 & & G & & 123 & & & & & II & & - \\
\hline 150 & 134452.71 & 49.96 & G & & 167 & & & & $2 \pm$ & II & 0.02 & - \\
\hline 151 & 134557.55 & & G & & 33 & 0.96 & & & 09 & II & & (2) \\
\hline 152 & 134600.30 & & G & & 15 & 1. & & & $<1.0$ & II & & - \\
\hline 153 & & & G & & 14 & & & & $<0.59$ & II & & - \\
\hline 154 & 13464 & & 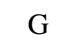 & & & & & & - & & & - \\
\hline 155 & & & G & & 21 & & & & & II & & - \\
\hline 156 & & & & & & & & & & & & - \\
\hline 157 & & & Q & & & & & & & II & & - \\
\hline 158 & & & Q & & 10 & & & & & & & - \\
\hline 159 & 134927.92 & 62015.11 & G & $42110 \pm 0.00010^{(\dagger)}$ & 131 & & & & 99 & II & & - \\
\hline 160 & & 62520.66 & G & & 220 & & & & $.72 \pm 0.09$ & II & $20.63 \pm 0.04$ & - \\
\hline 161 & 135414.72 & +491315.2 & G & $.43885 \pm 0.13902^{(\star)}$ & 123 & 0.72 & $633 \pm 127$ & $4.55 \pm 3.53$ & $0.91 \pm 0.09$ & II & $22.08 \pm 0.18$ & $\ldots$ \\
\hline
\end{tabular}


Table A.1. continued.

\begin{tabular}{|c|c|c|c|c|c|c|c|c|c|c|c|c|}
\hline Sr.No & $\begin{array}{c}\text { RA } \\
(\mathrm{HMS}) \\
(2)\end{array}$ & $\begin{array}{c}\text { Dec } \\
(\mathrm{DMS}) \\
(3)\end{array}$ & Class & (5) & $\begin{array}{l}\text { Size } \\
\left({ }^{\prime \prime}\right) \\
(6)\end{array}$ & $\begin{array}{l}\text { Size } \\
(\mathrm{Mpc}) \\
(7)\end{array}$ & $\begin{array}{c}S_{144 \mathrm{MHz}} \\
(\mathrm{mJy}) \\
(8)\end{array}$ & $\begin{array}{c}P_{144 \mathrm{MHz}} \\
\left(10^{26} \mathrm{~W} \mathrm{~Hz}^{-1}\right) \\
(9)\end{array}$ & $\begin{array}{l}\alpha_{144}^{1400} \\
(10)\end{array}$ & $\begin{array}{l}\text { FR } \\
\text { Type } \\
(11)\end{array}$ & $\begin{array}{c}r \\
\text { Mag } \\
(12)\end{array}$ & Ref. \\
\hline 102 & 50 & T2 & G & $2709 \pm 0.00011^{(\dagger)}$ & 291 & & & & & 11 & & - \\
\hline 163 & 5635.89 & 60944.77 & G & & 111 & 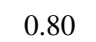 & & & $76 \pm 0.13$ & & & - \\
\hline 164 & 5951.16 & 70321.03 & G & $6224 \pm 0.00$ & 140 & 0.85 & & 03 & $55 \pm 0$ & II & .48 & - \\
\hline 165 & 140255.41 & 12728.60 & G & $1796+016062^{(\star)}$ & 135 & 0.87 & $304 \pm 61$ & $3.07=$ & $.79 \pm 0.09$ & $\mathrm{II}$ & $0.93 \pm 0.06$ & - \\
\hline 166 & 1 & 1 & G & & 228 & 1.41 & $1596 \pm 319$ & 2.00 & $87 \pm 0.09$ & 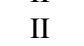 & 0.06 & 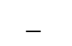 \\
\hline 167 & 61 & 16423 & G & $7 \quad 0 \Omega 60(\$)$ & 453 & 2.96 & & & - & I & $0.34 \pm 0.06$ & - \\
\hline 168 & 140540.97 & 541055.42 & G & 0 & 116 & & & & $2 \pm 0$ & II & & - \\
\hline 169 & 14060 & 54749.87 & G & $\star \star \star \star)$ & 114 & 0.8 & & 67 & $86 \pm 0.09$ & II & 0.40 & - \\
\hline 170 & 140612.30 & 533834.64 & G & $46455 \pm 0.00010^{(\dagger)}$ & 117 & 0.71 & $5 \pm 2$ & $0.04 \pm 0.02$ & $<0.55$ & II & & - \\
\hline 171 & 140718.48 & +513204.63 & G & & 177 & 0.89 & $246 \pm 849$ & $16.50 \pm 3.31$ & $83 \pm 0.09$ & II & & - \\
\hline 172 & 14071 & +55060 & G & & 180 & & & & - & II & & - \\
\hline 173 & 14083 & & Q & & 85 & & & & & H & & - \\
\hline 174 & 1 & & G & & 240 & & & & & & & 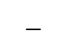 \\
\hline 175 & 1411 & 47 & G & & 30 & & & & & & & - \\
\hline 176 & 97 & & O & & 99 & & & & & & & - \\
\hline 177 & & & & & & & & & & & & - \\
\hline 178 & & & G & & & & & & & & & - \\
\hline 179 & & & Q & & 111 & & & & & $\mathrm{I}$ & & (2) \\
\hline 180 & & & G & & 117 & & & & & II & & - \\
\hline 181 & & & G & & 336 & & & & & II & & - \\
\hline 182 & & & G & & 134 & & & & & $\mathrm{H}$ & & - \\
\hline 183 & 39 & 66 & G & & 142 & 1. & & & & II & & - \\
\hline 184 & 1419 & 22 & Q & (†) & 127 & 0 & & & & II & & - \\
\hline 185 & 1420 & & G & (†) & 101 & & & & & II & & - \\
\hline 186 & 1 & & G & & & & & & & - & & - \\
\hline 187 & & & G & & & & & & - & 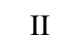 & & - \\
\hline 188 & & & Q & & & & & & - & 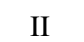 & & - \\
\hline 18 & & & & & & & & & & & & - \\
\hline 19 & & & $\mathrm{C}$ & & & & & & & & & - \\
\hline 191 & & & G & & 1 & & & & & II & & - \\
\hline 192 & & & G & & 20 & & & & & II & & - \\
\hline 193 & & & G & & 130 & & & & & III & & - \\
\hline 194 & & & G & (†) & 452 & 1. & & & & II & & - \\
\hline 195 & & & 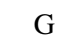 & & 280 & & & & & II & & - \\
\hline 196 & & & a & & 40 & & & & & II & & - \\
\hline 197 & 55 & & G & & & & & & & II & & - \\
\hline 198 & 143136.99 & 2724.90 & G & $7^{(\dagger)}$ & 186 & & & & 99 & II & & _ \\
\hline 199 & & & Q & & 91 & & & & - & II & & - \\
\hline 200 & & & G & & & & & & & II & & - \\
\hline 201 & & & Q & & & & & & - & to & & - \\
\hline 202 & & & 0 & & & & & & & & & (10) \\
\hline 203 & & & 0 & & & & & & & & & - \\
\hline 204 & & & & & & & & & & th & & - \\
\hline 205 & & & G & & 207 & $x^{2}+2>$ & & & 9 & II & & - \\
\hline 206 & 03 & 20 & 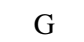 & $(\dagger)$ & 115 & 0.8 & $8=$ & & 16 & II & .10 & - \\
\hline 207 & & 552706.08 & $C_{1}$ & $(\star)$ & 238 & & & & 0 & II & & - \\
\hline 208 & & 02601.87 & Q & (†) & 143 & & & $J 0 \pm 4.49$ & $0.96 \pm 0.10$ & II & & - \\
\hline 209 & 144017.49 & 532620.29 & G & $2672 \pm 0.00013^{(\dagger)}$ & 195 & 1.26 & \pm 70 & $0 \pm 0.72$ & - & II & $3 \pm 0.04$ & - \\
\hline 210 & 144215.00 & 470646.09 & G & $0.44503 \pm 0.00011^{(\dagger)}$ & 121 & 0 . & - & & \pm( & II & & - \\
\hline 211 & 4258.59 & +520902.07 & G & $0.272 \pm 0.057^{(\S)}$ & 289 & 1. & 82 & 2 & $.88 \pm 0.09$ & II & & - \\
\hline 212 & 14430 & +511810.01 & $\mathrm{G}$ & $46603 \pm 0.00011^{(\dagger)}$ & 15 & 0. & & & $<1.10$ & II & & - \\
\hline 213 & 14433270 & +454654.56 & G & & 11 & & & & & II & & - \\
\hline 21 & & & Q & & 19 & & & & & II & & - \\
\hline 215 & 14441 & & $F_{2}+2$ & & 14 & & & & & F & & - \\
\hline 216 & & & G & & 16 & & & & & III & & - \\
\hline 217 & & & G & & 16 & & & & & & & - \\
\hline 218 & 144607.20 & 484137.79 & G & $0.37676 \pm 0.00006^{(\dagger)}$ & 146 & 0.78 & $174 \pm 35$ & $0.87 \pm 0.17$ & $0.89 \pm 0.09$ & II & $19.10 \pm 0.02$ & - \\
\hline
\end{tabular}


Table A.1. continued.

\begin{tabular}{|c|c|c|c|c|c|c|c|c|c|c|c|c|}
\hline Sr.No & $\begin{array}{c}\text { RA } \\
(\mathrm{HMS}) \\
(2) \\
\end{array}$ & $\begin{array}{c}\text { Dec } \\
(\mathrm{DMS}) \\
(3)\end{array}$ & Class & (5) & $\begin{array}{l}\text { Size } \\
\left({ }^{\prime \prime}\right) \\
(6) \\
\end{array}$ & $\begin{array}{l}\text { Size } \\
(\mathrm{Mpc}) \\
(7)\end{array}$ & $\begin{array}{c}S_{144 \mathrm{MHz}} \\
(\mathrm{mJy}) \\
(8)\end{array}$ & $\begin{array}{c}P_{144 \mathrm{MHz}} \\
\left(10^{26} \mathrm{~W} \mathrm{~Hz}^{-1}\right) \\
(9)\end{array}$ & $\begin{array}{l}\alpha_{144}^{1400} \\
(10) \\
\end{array}$ & $\begin{array}{l}\text { FR } \\
\text { Type } \\
(11) \\
\end{array}$ & $\begin{array}{c}r \\
\text { Mag } \\
(12) \\
\end{array}$ & \\
\hline 219 & 88 & $36 ?$ & G & $4 \pm 0$ & 27 & 1. & & & & II & & - \\
\hline 220 & 82 & & 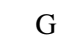 & & . & 0. & & & & III & & - \\
\hline 221 & 36 & & G & 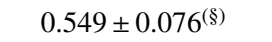 & 26 & 0.8 & & & 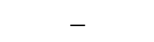 & II & & - \\
\hline 222 & 28 & & Q & 00 & 184 & 1.48 & 1407 & & $1+e^{2}$ & II & & - \\
\hline 223 & 41 & 34 & Q & †) & 246 & 1.43 & & & $0.80 \pm 0$ & II & & (2) \\
\hline 224 & 59 & 28 & G & & 194 & 1.23 & & & - & II & & - \\
\hline 225 & 28 & 5 & G & & 208 & 1.3 & & & 0 & II & & - \\
\hline 226 & 145250.73 & +51370 & G & 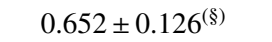 & 108 & 0.7 & & & 1.0 & II & & - \\
\hline 227 & 145702.81 & +480646.64 & G & $26720+000$ & 168 & 0.7 & & & $<1.11$ & II & & - \\
\hline 228 & 145749.59 & 62 & G & $0620,0146(8)$ & 199 & 1.4 & 55 & & 88 & II & & - \\
\hline 229 & 150000.56 & +504355.62 & G & $0.415 \pm 0.0$ & 142 & & & & & II & & - \\
\hline 230 & & & G & $2 \pm 0$. & 164 & & & & & W & & - \\
\hline 231 & & & G & & & & & & & & & - \\
\hline 232 & & & G & & & & & & & II & & - \\
\hline 233 & 61 & 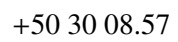 & G & 65. & 20 & 1 & 00 & 23 & $0.91 \pm$ & II & .17 & - \\
\hline 234 & 81 & 11 & G & $1 \pm 0$ & 26 & 1. & 57 & 9 & - & II & 05 & - \\
\hline 235 & 150624.10 & 61 & G & $0437 \pm 0$ & 184 & 0.85 & 24 & 7 & $7 \pm 0$ & II & 17. & - \\
\hline 236 & & .03 & G & 0700 & 105 & 0.7 & & & $0.95 \pm$ & 11 & $21.84 \pm 0.13$ & - \\
\hline 237 & & 70 & G & $9 \pm 0$ & 119 & & 74 & & $0.77 \pm$ & II & $21.14 \pm$ & - \\
\hline 238 & 151845.36 & 523708.56 & G & $52105 \pm 0.00011^{(\dagger)}$ & 110 & & & & $1.11 \pm 0.09$ & II & $20.24 \pm 0.05$ & - \\
\hline 239 & 152311.09 & +520303.52 & Q & $0.51667 \pm 0.00010^{(\dagger)}$ & 132 & 0.84 & $382 \pm 77$ & $4.35 \pm 0.87$ & $1.09 \pm 0.09$ & II & $20.74 \pm 0.06$ & - \\
\hline
\end{tabular}

Table A.2. LoTSS GRGs in galaxy clusters.

\begin{tabular}{|c|c|c|c|c|c|c|c|c|}
\hline No & Cluster name & GRG RA & GRG Dec & $z$ & $\begin{array}{c}r_{200} \\
(\mathrm{Mpc})\end{array}$ & $R_{L *}$ & $\begin{array}{c}M_{200} \\
\left(10^{14} M_{\odot}\right)\end{array}$ & $N_{200}$ \\
\hline 1 & WHL J112126.4+534457 & 112126.44 & +534456.71 & $0.10378 \pm 0.00002$ & 1.7 & 69.4 & 4.6 & 46 \\
\hline 2 & WHL J113250.7+505705 & 113250.67 & +505704.68 & $0.35857 \pm 0.00010$ & 0.9 & 16.9 & 0.9 & 14 \\
\hline 3 & WHL J113503.2+482612 & 113503.20 & +482612.12 & $0.22597 \pm 0.00006$ & 1.1 & 21.7 & 1.2 & 17 \\
\hline 4 & WHL J121900.8+505255 & 121900.76 & +505254.41 & $0.38509 \pm 0.00008$ & 0.9 & 17.3 & 0.9 & 14 \\
\hline 5 & GMBCG J188.75636+53.29864 & 123501.52 & +531755.09 & $0.34480 \pm 0.00030$ & - & - & - & - \\
\hline 6 & GMBCG J191.35486+47.11948 & 124525.16 & +470710.11 & $0.51179 \pm 0.00015$ & - & - & - & - \\
\hline 7 & WHL J124913.2+500044 & 124913.20 & +500043.68 & $0.34956 \pm 0.00010$ & 1.1 & 25.3 & 1.4 & 30 \\
\hline 8 & WHL J125142.1+503425 & 125142.04 & +503424.65 & $0.54904 \pm 0.00007$ & 1.1 & 24.3 & 1.4 & 11 \\
\hline 9 & WHL J131404.6+543938 & 131404.60 & +543937.88 & $0.34645 \pm 0.00008$ & 1.2 & 26.1 & 1.5 & 23 \\
\hline 10 & WHL J132229.1+504845 & 132229.07 & +504844.53 & $0.18439 \pm 0.00004$ & 0.9 & 15.9 & 0.8 & 11 \\
\hline 11 & WHL J133258.3+535356 & 133258.28 & +535355.60 & $0.32261 \pm 0.00009$ & 0.9 & 16.6 & 0.9 & 13 \\
\hline 12 & WHL J133512.0+494427 & 133512.01 & +494427.20 & $0.436 \pm 0.041^{(\dagger)}$ & 0.9 & 14.7 & 0.7 & 9 \\
\hline 13 & WHL J133618.8+533952 & 133618.75 & +533952.12 & $0.30138 \pm 0.00006$ & 0.9 & 13.4 & 0.7 & 11 \\
\hline 14 & WHL J134207.0+472553 & 134206.98 & +472553.04 & $0.17213 \pm 0.00004$ & 0.9 & 13.2 & 0.7 & 13 \\
\hline 15 & WHL J142857.4+542353 & 142857.40 & +542352.99 & $0.40573 \pm 0.00011$ & 1.2 & 30.7 & 1.8 & 22 \\
\hline 16 & WHL J142933.4+544336 & 142933.45 & +544335.29 & $0.12328 \pm 0.00002$ & 1.3 & 34.7 & 2.0 & 29 \\
\hline 17 & WHL J144410.5+554746 & 144410.50 & +554745.64 & $0.33475 \pm 0.00005$ & 1.2 & 30.1 & 1.7 & 26 \\
\hline 18 & WHL J144520.9+540330 & 144520.87 & +540329.62 & $0.43345 \pm 0.00013$ & 1.0 & 25.1 & 1.4 & 18 \\
\hline 19 & GMBCG J222.81919+51.22588 & 145116.59 & +511333.28 & $0.509 \pm 0.050^{(\dagger)}$ & - & - & - & - \\
\hline 20 & WHL J150624.1+535503 & 150624.10 & +535502.61 & $0.30437 \pm 0.00005$ & 1.0 & 19.1 & 1.0 & 11 \\
\hline
\end{tabular}

Notes. Parameters $r_{200}$ and $R_{L^{*}}$ (cluster richness parameter) have been taken from the WHL galaxy cluster catalogue and $M_{200}$ was computed from Eq. (2) of Wen et al. (2012). GRGs marked with ${ }^{(\dagger)}$ in the redshift column have photometric redshift estimate and the rest have spectroscopic redshifts. $r_{200}$ is the radius within which the mean density of a cluster is 200 times of the critical density of the Universe, and $M_{200}$, is the mass of the cluster within $r_{200}$. $N_{200}$ is the number of galaxies within $r_{200}$. Clusters 6, 7, and 20 are identified from GMBCG cluster catalogue (Hao et al. 2010). 


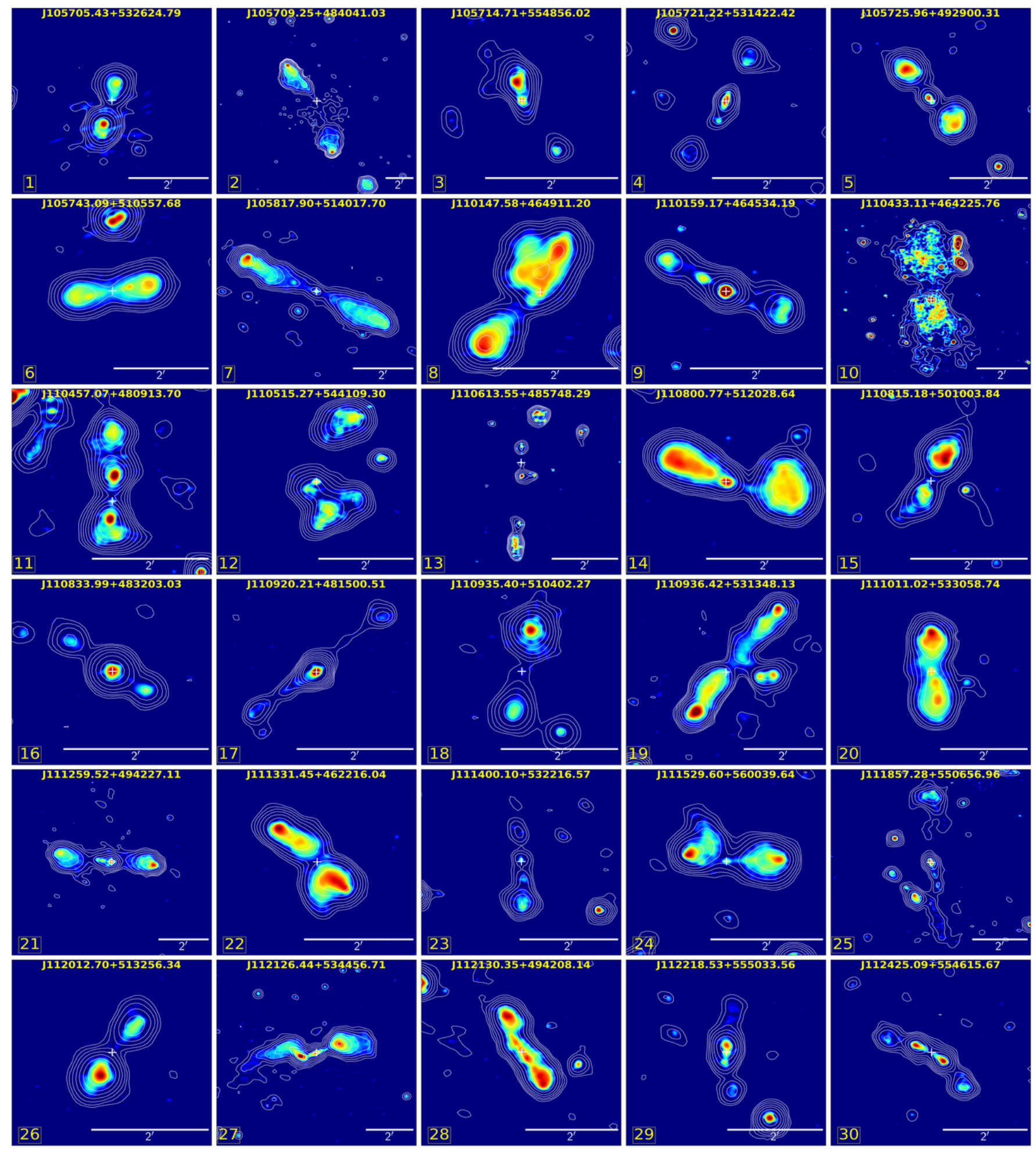

Fig. A.1. LoTSS high-resolution $\left(\sim 6^{\prime \prime}\right)$ images 1-30 with LoTSS low-resolution $\left(\sim 20^{\prime \prime}\right)$ contours overlaid. The cross "+" marker indicates the location of the host galaxy. The radio contours are drawn with eight levels, which are chosen by equally (log scale) dividing the data value range above $\sim 3 \sigma$, where $\sigma$ is the local rms of the map. 
P. Dabhade et al.: GRGs in LoTSS DR1

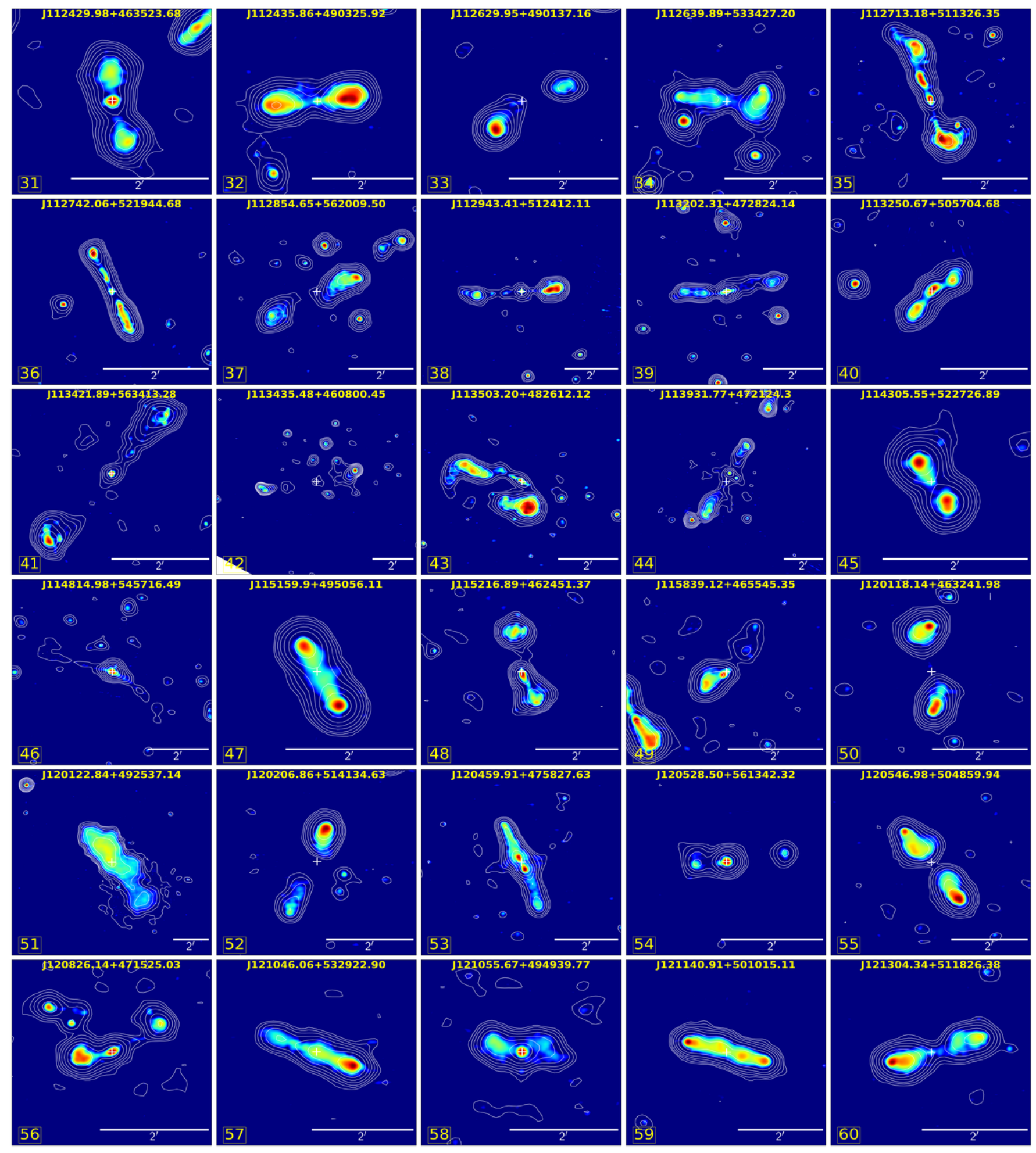

Fig. A.2. LoTSS high-resolution $\left(\sim 6^{\prime \prime}\right)$ images 31-60 with LoTSS low-resolution $\left(\sim 20^{\prime \prime}\right)$ contours overlaid.The cross "+" marker indicates the location of the host galaxy. The radio contours are drawn with eight levels, which are chosen by equally (log scale) dividing the data value range above $\sim 3 \sigma$, where $\sigma$ is the local rms of the map. 
A\&A 635, A5 (2020)

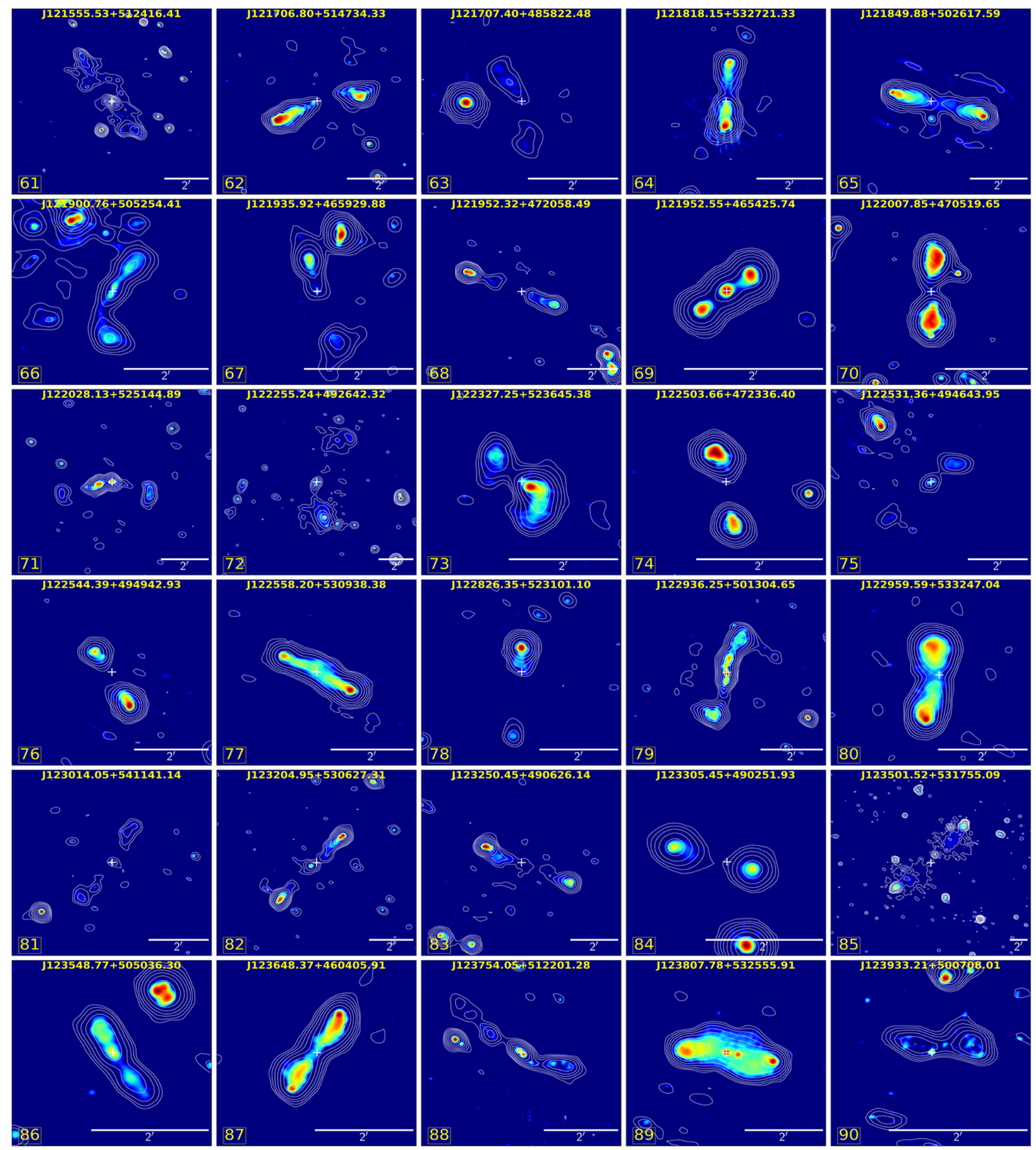

Fig. A.3. LoTSS high-resolution $\left(\sim 6^{\prime \prime}\right)$ images 61-90 with LoTSS low-resolution $\left(\sim 20^{\prime \prime}\right)$ contours overlaid. The cross "+" marker indicates the location of the host galaxy. The radio contours are drawn with eight levels, which are chosen by equally (log scale) dividing the data value range above $\sim 3 \sigma$, where $\sigma$ is the local rms of the map. 
P. Dabhade et al.: GRGs in LoTSS DR1

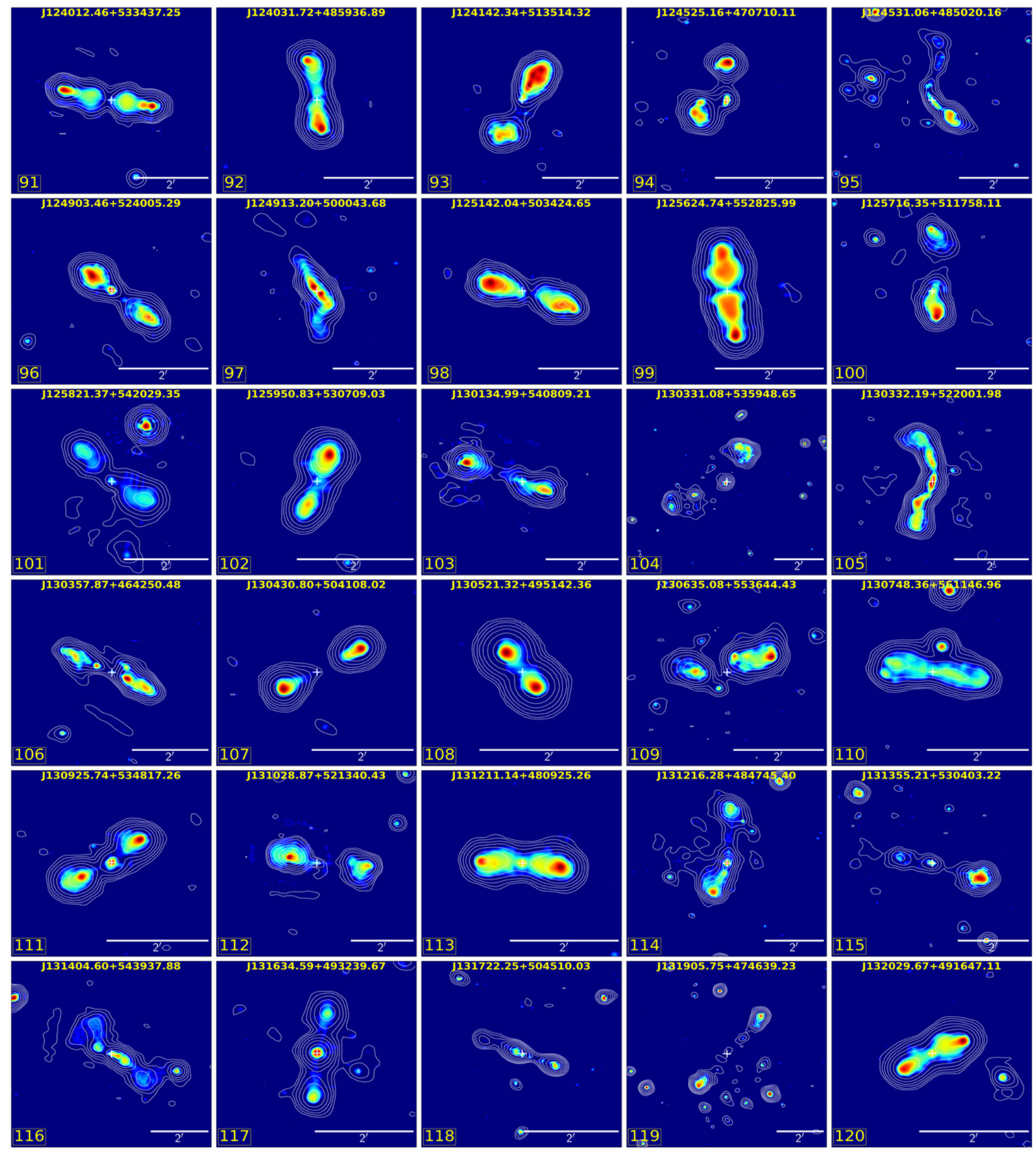

Fig. A.4. LoTSS high-resolution $\left(\sim 6^{\prime \prime}\right)$ images $91-120$ with LoTSS low-resolution $\left(\sim 20^{\prime \prime}\right)$ contours overlaid. The cross "+" marker indicates the location of the host galaxy. The radio contours are drawn with eight levels, which are chosen by equally (log scale) dividing the data value range above $\sim 3 \sigma$, where $\sigma$ is the local rms of the map. 

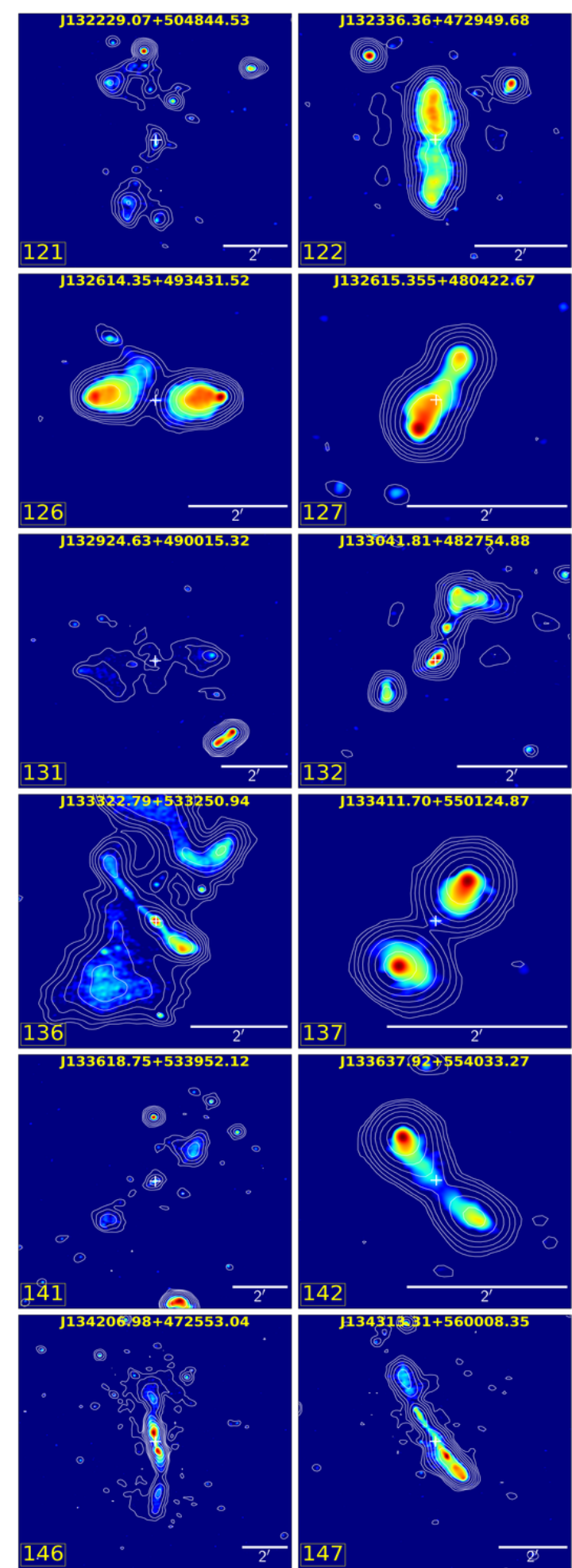
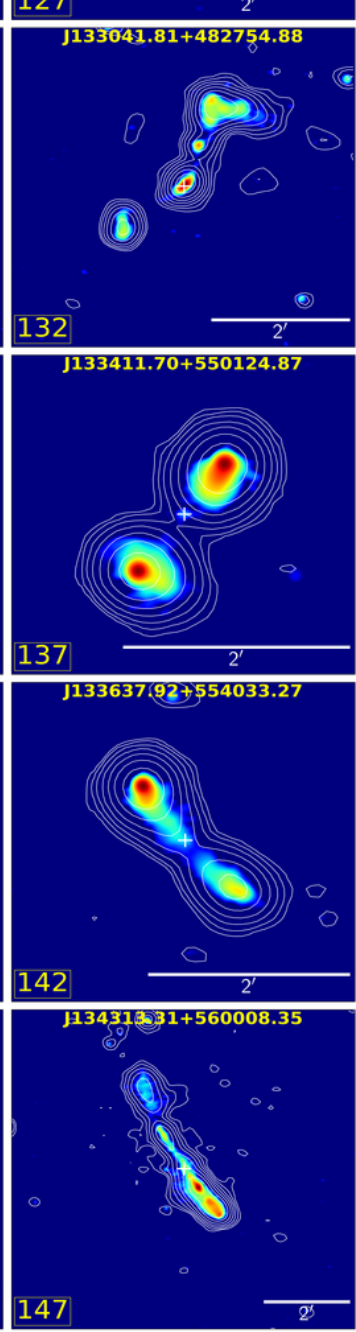
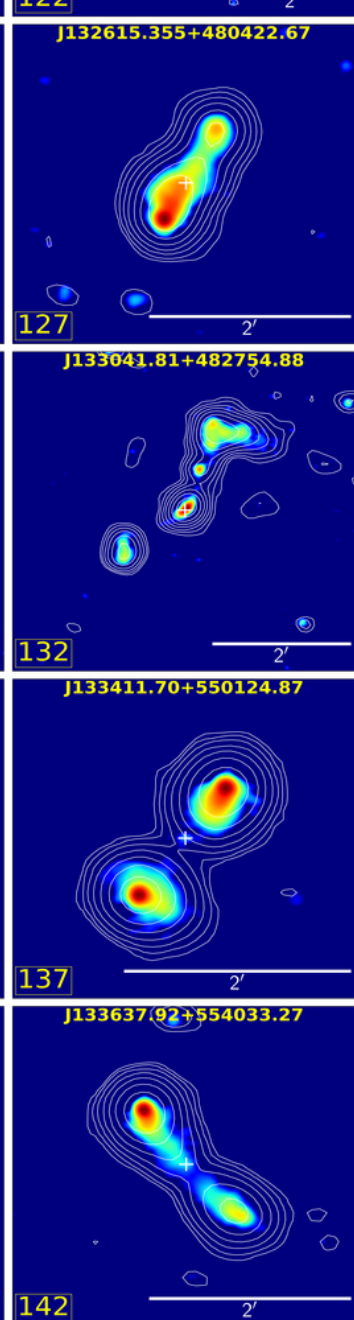
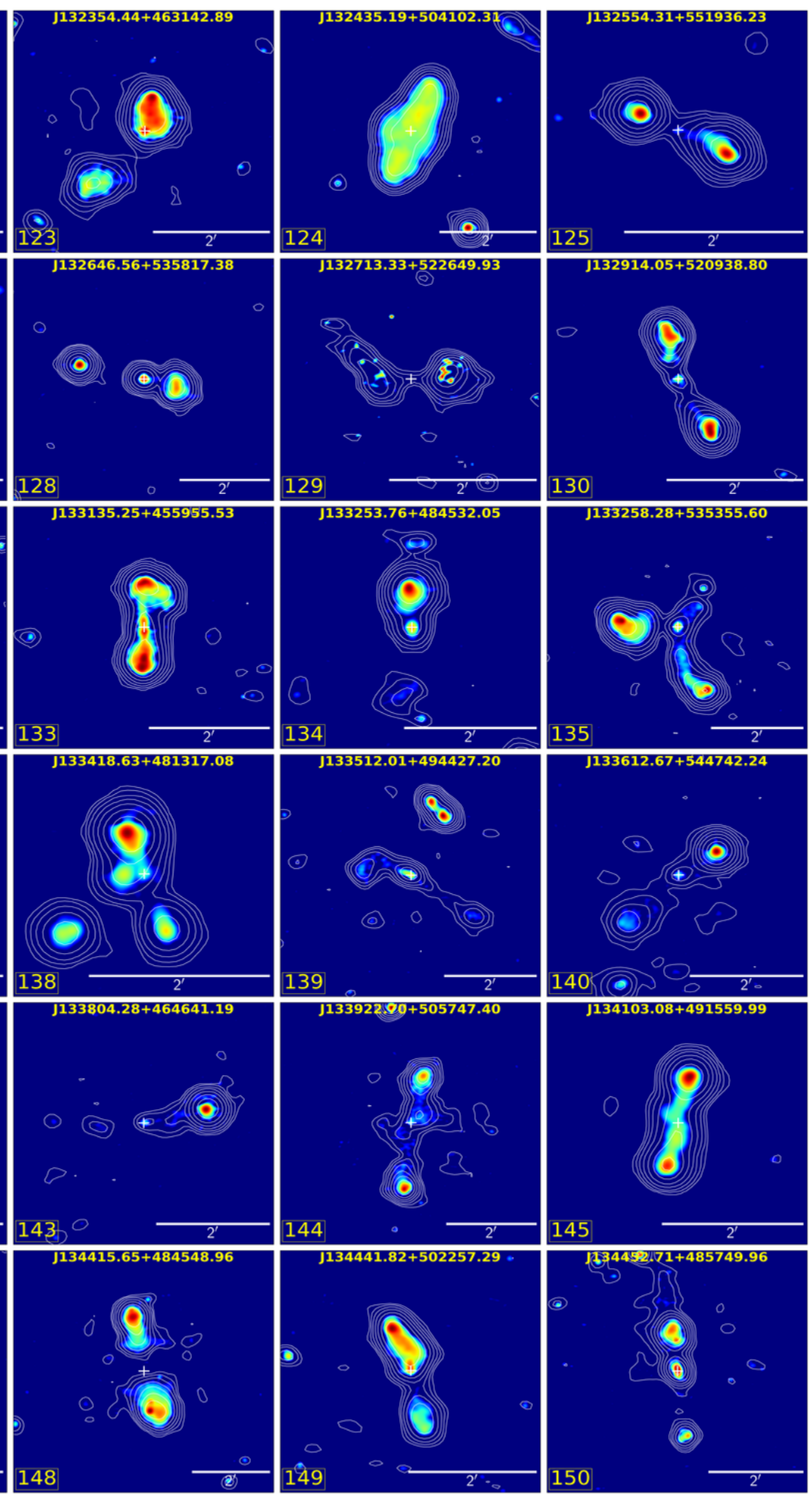

Fig. A.5. LoTSS high-resolution $\left(\sim 6^{\prime \prime}\right)$ images $121-150$ with LoTSS low-resolution $\left(\sim 20^{\prime \prime}\right)$ contours overlaid. The cross "+" marker indicates the location of the host galaxy. The radio contours are drawn with eight levels, which are chosen by equally (log scale) dividing the data value range above $\sim 3 \sigma$, where $\sigma$ is the local rms of the map. 
P. Dabhade et al.: GRGs in LoTSS DR1

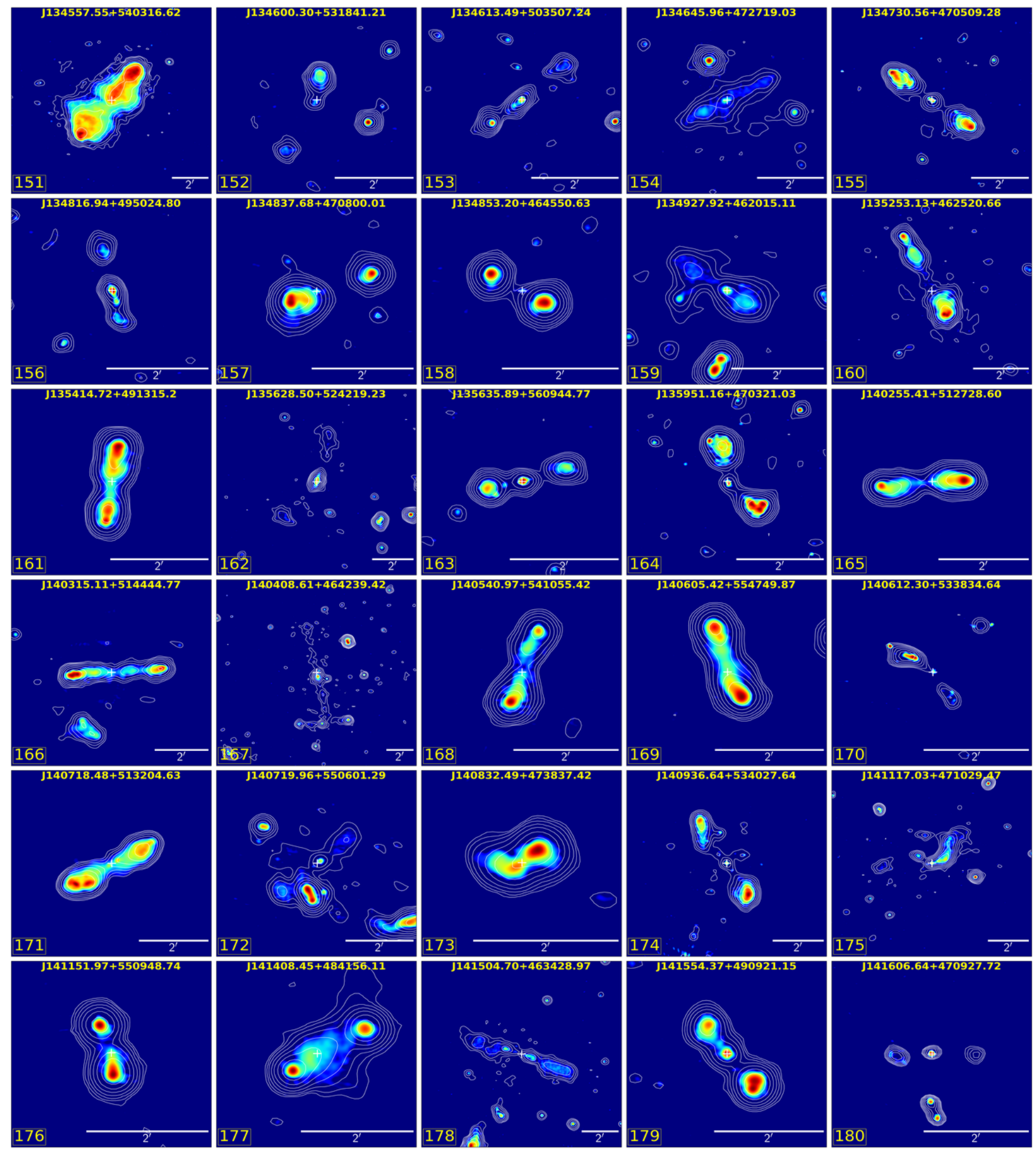

Fig. A.6. LoTSS high-resolution $\left(\sim 6^{\prime \prime}\right)$ images 151-180 with LoTSS low-resolution $\left(\sim 20^{\prime \prime}\right)$ contours overlaid. The cross "+" marker indicates the location of the host galaxy. The radio contours are drawn with eight levels, which are chosen by equally (log scale) dividing the data value range above $\sim 3 \sigma$, where $\sigma$ is the local rms of the map. 
A\&A 635, A5 (2020)

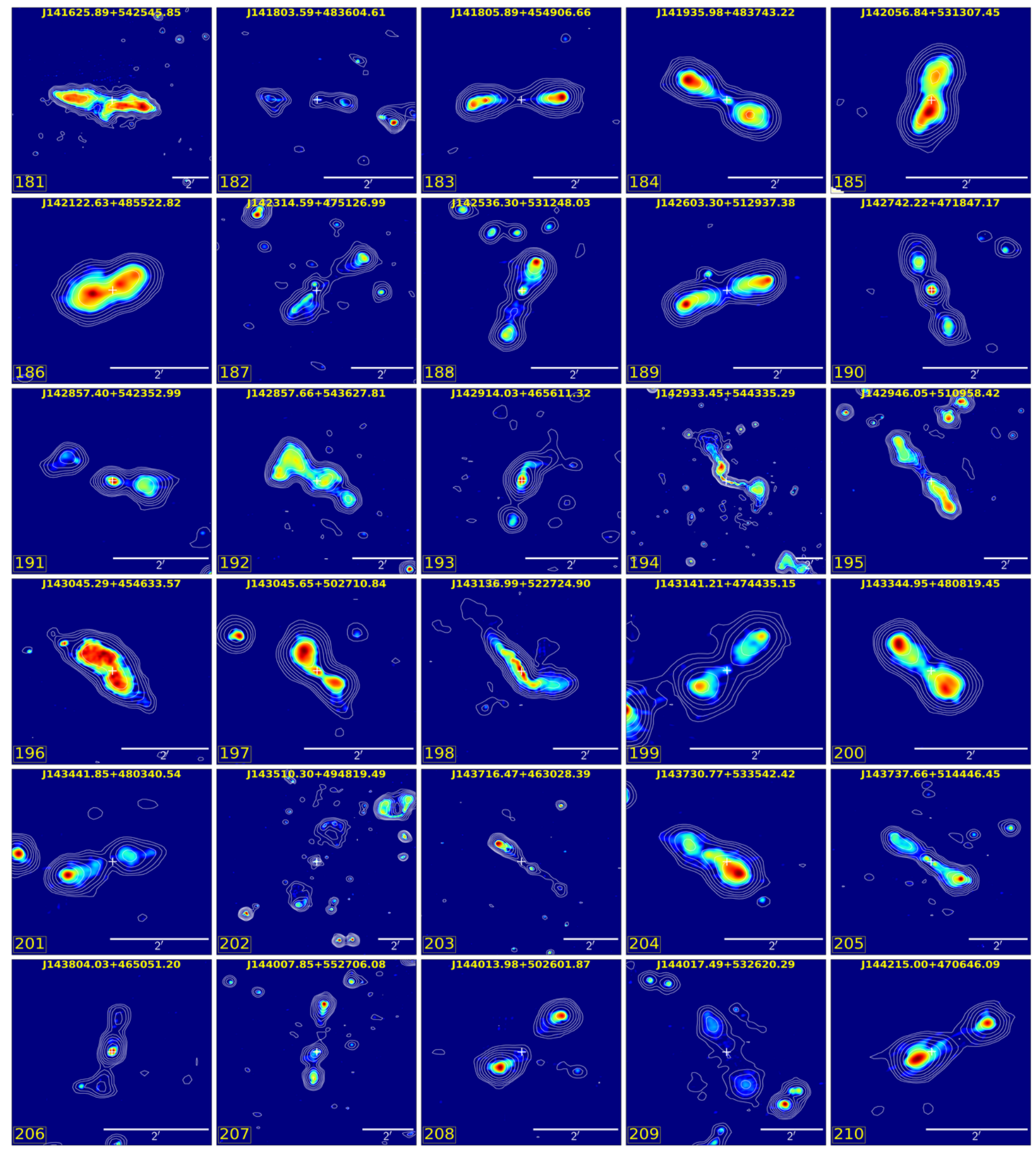

Fig. A.7. LoTSS high-resolution $\left(\sim 6^{\prime \prime}\right)$ images 181-210 with LoTSS low-resolution $\left(\sim 20^{\prime \prime}\right)$ contours overlaid. The cross “+” marker indicates the location of the host galaxy. The radio contours are drawn with eight levels, which are chosen by equally (log scale) dividing the data value range above $\sim 3 \sigma$, where $\sigma$ is the local rms of the map. 
P. Dabhade et al.: GRGs in LoTSS DR1

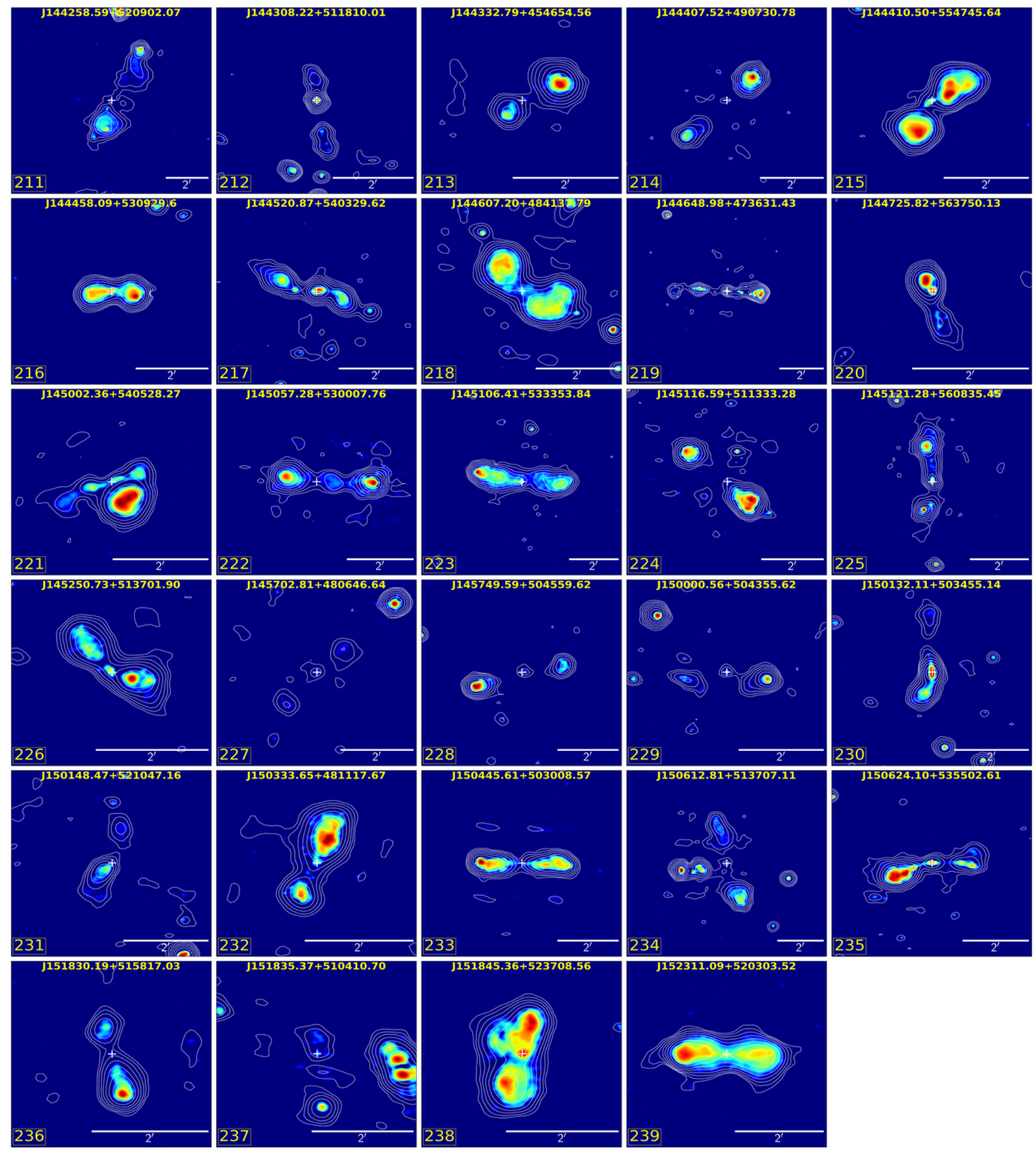

Fig. A.8. LoTSS high-resolution $\left(\sim 6^{\prime \prime}\right)$ images 211-239 with LoTSS low-resolution $\left(\sim 20^{\prime \prime}\right)$ contours overlaid. The cross "+" marker indicates the location of the host galaxy. The radio contours are drawn with eight levels, which are chosen by equally (log scale) dividing the data value range above $\sim 3 \sigma$, where $\sigma$ is the local rms of the map. 